\title{
Gradhiva
}

GRADHIV

Revue d'anthropologie et d'histoire des arts

19 | 2014

"L'Atlantique Noir » de Nancy Cunard. Negro Anthology 1931-1934

\section{Paul Robeson et la représentation des Noirs dans le cinéma de l'entre-deux-guerres. Primitivisme et double conscience}

Paul Robeson and the representation of Black Americans in films during the interwar period. Primitivism and double-consciousness

\section{François Bovier}

\section{OpenEdition}

Journals

Édition électronique

URL : http://journals.openedition.org/gradhiva/2801

DOI : 10.4000/gradhiva.2801

ISSN : 1760-849X

Éditeur

Musée du quai Branly Jacques Chirac

\section{Édition imprimée}

Date de publication : 1 mars 2014

Pagination : 132-159

ISBN : 978-2-35744-073-9

ISSN : 0764-8928

\section{Référence électronique}

François Bovier, «Paul Robeson et la représentation des Noirs dans le cinéma de l'entre-deux-guerres. Primitivisme et double conscience », Gradhiva [En ligne], 19 | 2014, mis en ligne le 01 mars 2017, consulté le 05 mai 2019. URL : http://journals.openedition.org/gradhiva/2801 ; DOI : 10.4000/ gradhiva.2801 


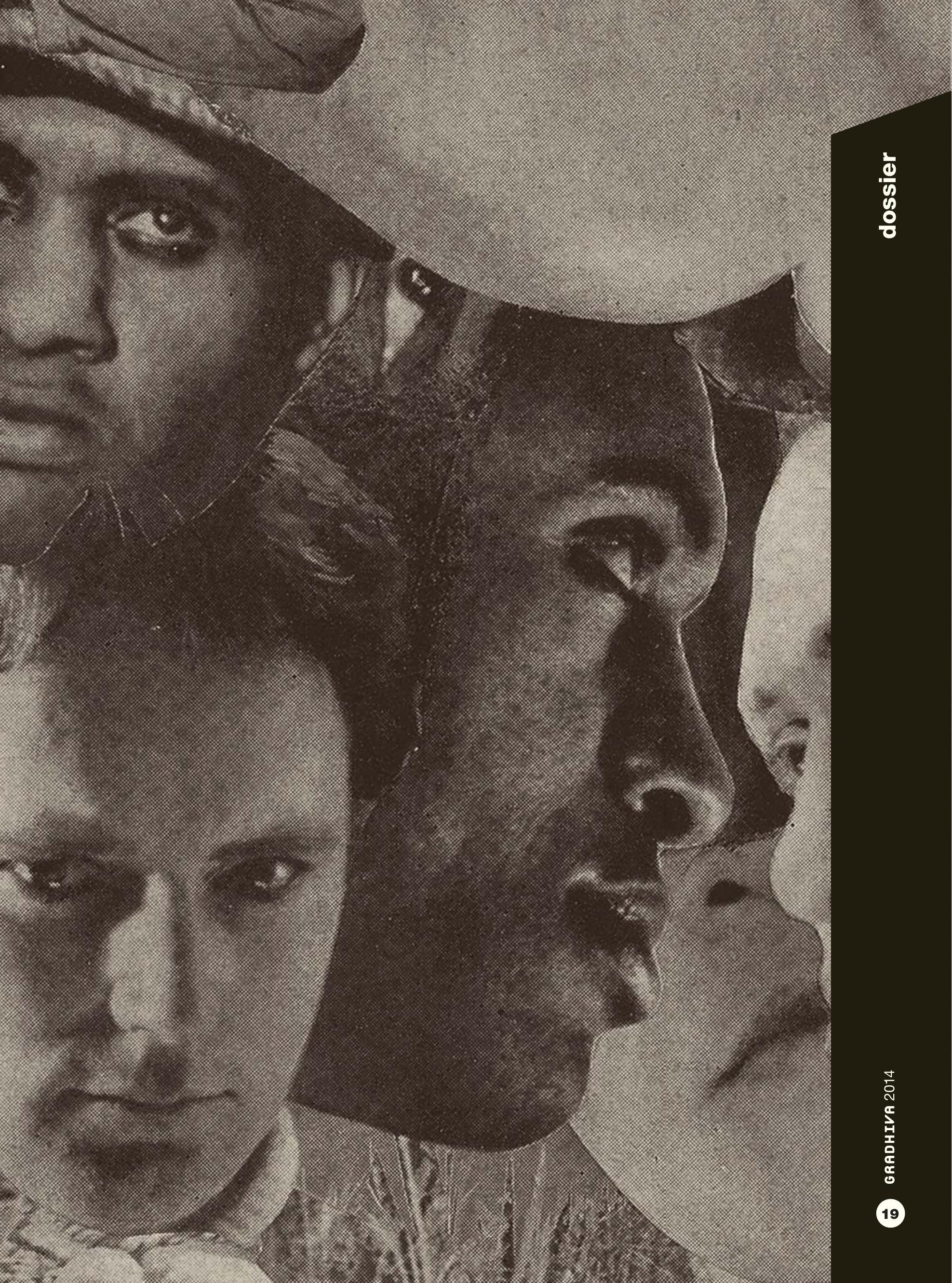




\section{Paul Robeson et la représentation des Noirs dans le cinéma de l'entre-deux-guerres}

Primitivisme et double conscience

par François Bovier

La représentation des Noirs au cinéma répond à des stéréotypes avilissants, en usage dès les années 1910. Les premiers rôles de Paul Robeson, acteur et chanteur charismatique associé à la Renaissance de Harlem, problématisent cette logique raciste. Il joue pour la première fois à l'écran dans une production intégralement noire d'Oscar Micheaux (Body and Soul, USA, 1925) qui stigmatise l'ambivalence des pièces de théâtre auxquelles Robeson est associé et les paradoxes de la double-conscience qu'il incarne. Dans le film d'avant-garde Borderline (Kenneth Macpherson, Suisse, 1930), Robeson négocie une image apparemment progressiste de l'Afro-Américain, laquelle, malgré le texte programmatique appelant à l'avènement d'un cinéma authentiquement noir que Macpherson écrit pour le recueil de Nancy Cunard, répond en fait aux fantasmes primitivistes du cinéaste. 


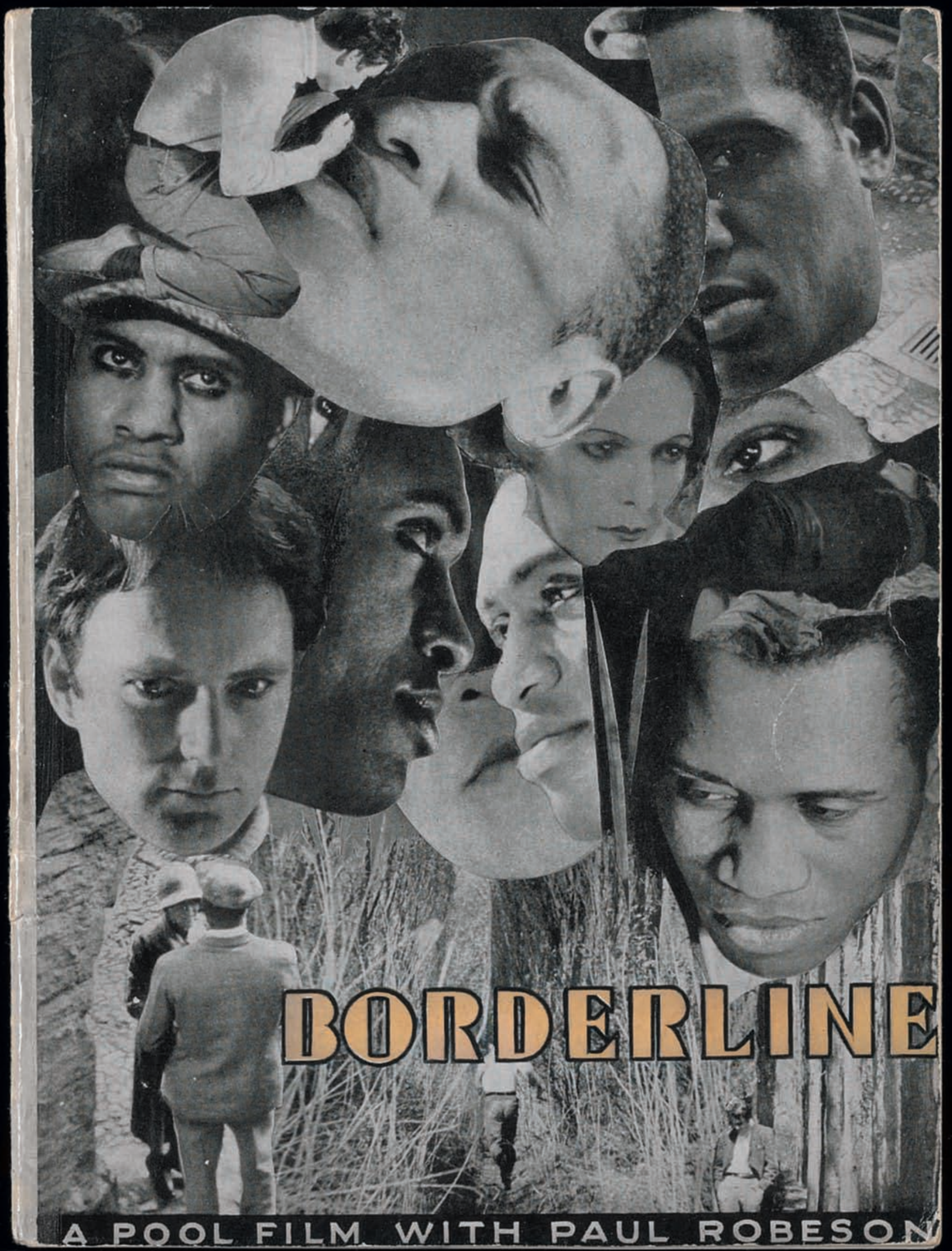


Le théâtre, la musique, la littérature et, dans une moindre mesure, les arts visuels constituent dans les années 1920-1930 les principaux supports d'expression de la culture afro-américaine ${ }^{\mathbf{1}}$, indissociable des revendications d'égalité raciale. Parallèlement à l'activité militante d'associations de lutte pour les droits civiques telles que la National Association for the Advancement of Colored People (NAACP) et son journal The Crisis ${ }^{2}$ (qui atteint un tirage de 100000 exemplaires au début des années 1920), certaines publications influentes participent à la reconnaissance des droits fondamentaux des Africains-Américains. C'est le cas de l'anthologie d'Alain Locke parue en 1925, The New $\mathrm{Negro}^{3}$, qui popularise la culture de la Renaissance de Harlem et la volonté d'émancipation de ses protagonistes. L'anthologie «fleuve» (pas moins de 855 pages) que Nancy Cunard édite entre 1931 et 1933, Negro Anthology, s'inscrit dans la continuité de l'ouvrage pionnier de Locke: des études historiques sur l'esclavage ou le racisme, sur les droits des Afro-Américains ou le scandale judiciaire de Scottsboro, des essais sur la littérature, la poésie, la musique, la sculpture et la danse côtoient des poèmes et des témoignages plus personnels. Parallèlement à ces publications, le cinéma et la photographie représentent, en tant que moyens de reproduction de masse, des porte-voix privilégiés pour véhiculer une image du «nouveau Nègre» en rupture avec les stéréotypes avilissants encore majoritaires dans la pensée et la culture occidentales.

Aussi n'est-il pas surprenant de trouver dans l'anthologie de Cunard un article qui porte sur la question de la représentation des Noirs au cinéma, sous la plume de Kenneth Macpherson. «A Negro Film Union - Why not ${ }^{4}$ ? » constitue une forme de manifeste pour un cinéma de race authentique, dont l'argumentation peut paraître ambivalente - Macpherson ne manque pas, d'ailleurs, de préciser que sa position de cinéaste occidental est équivoque à cet égard. Cette situation d'extériorité raciale est cependant caractéristique de l'anthologie coordonnée par Cunard, qui intègre des contributions de Noirs et de Blancs; plus généralement, cette interaction constitue l'une des spécificités de la Renaissance de Harlem, scène artistique et politique en quête d'une légitimité culturelle ${ }^{5}$. Robert Herring, critique de films, collaborateur régulier de la revue Close Up et éditeur littéraire (notamment de la revue Life and Letters Today), fait le lien entre Cunard et Macpherson: c'est lui qui introduit Macpherson à la scène de la Renaissance de Harlem et lui présente le célèbre acteur et chanteur Paul Robeson ${ }^{6}$. Macpherson éprouve une véritable fascination pour la culture afro-américaine qu'il exprime, en dehors de sa vie privée ${ }^{7}$, à travers son activité de cinéaste et d'éditeur au sein du groupe Pool, actif de 1927 à 1933. Plateforme d'édition et structure de production de films, Pool est fondé par un singulier ménage à trois, à savoir Macpherson, Bryher (pseudonyme d'Annie Winifred Ellerman, héritière d'une grande fortune britannique propriétaire d'une compagnie de bateaux, à l'instar de Cunard) et H.D. (nom de plume de la poétesse américaine Hilda Doolittle). En août 1929, Macpherson consacre un numéro de la revue Close Up à la représentation des Noirs à l'écran ${ }^{\mathbf{8}}$ - dossier tout à fait remarquable au vu de la carence de la littérature secondaire sur le sujet et du caractère marginal de cette problématique dans les milieux de la cinéphilie qui constituent le principal lectorat de Close Up. En 1930, il réalise de façon indépendante un long-
1. Dans le contexte de la Renaissance de Harlem, c'est avant tout le théâtre qui médiatise la culture afroaméricaine (Lewis 1981: 91).

2. Sur la NAACP et son organe officiel The Crisis (fondé en 1910), voir Jonas 2005.

3. Cette anthologie de fictions, poésie, théâtre et essais critiques, sociologiques, ethnologiques et historiques légitime la spécificité de la culture afro-américaine (notamment en reproduisant des œuvres d'art et des partitions de musique) et prône l'intégration des Noirs à la société américaine, tout en dénonçant la domination du monde occidental.

\section{Macpherson 1934.}

5. Voir notamment Baker 1987 ; Hutchinson 1995.

\section{page précédente} et ci-contre

fig. 1

H. D. (Hilda Doolittle), Borderline, Pool film, avec Paul Robeson, 1930. Yale Collection of American Literature, Yale University Beinecke Rare Books \& Manuscript Library, Za D721 930B (C) H.D. Estate.
6. Debo 2012: 154-155. Robeson est d'abord reconnu en tant qu'acteur, en association avec la compagnie Provincetown Players : en 1924, il interprète avec succès deux pièces d'Eugene O'Neill, The Emperor Jones et All God's Chillun Got Wings. En avril 1925, il donne au Greenwich Village Theater un récital en solo qui a fait date, à partir d'un répertoire exclusivement issu de la culture noire (McGinty et Shirley 1998: 105-106). La même année, il joue dans Body and Soul d'Oscar Micheaux - expérience qu'il considère comme désastreuse, évitant de mentionner ce film dans ses écrits et interviews (Musser 1998: 82)

7. Macpherson entretient des liaisons avec de jeunes Noirs, l'un d'entre eux habitant à la villa Kenwin, en Suisse, où vivent Macpherson, H.D. et Bryher, les trois membres fondateurs de Pool, au début des années 1930.

8. Macpherson et Bryher (éd.) 1929: 85-165 (avec des contributions de Macpherson, Geraldyn Dismond, Robert Herring, Walter White, Pau Green, Harry A. Potamkin, Elmer Anderson Carter, Oswell Blakeston, Hay Chowl). 
9. Sur les liens ambivalents entre le primitivisme et le modernisme, voir notamment Flam et Deutch (éd.) 2003; Rubin (éd.) 1984

10. Dans The Souls of Black Folk, W.E.B. Du Bois décrit le phénomène de la «double conscience" qui surdétermine les mécanismes de la

ségrégation aux États-Unis : "C'est une sensation bizarre, cette double conscience, ce sentiment de constamment se regarder par les yeux d'un autre, de mesurer son âme à l'aune d'un monde qui vous considère comme un spectacle avec un amusement teinté de pitié méprisante. " (Du Bois 2007 : 9) Certains films d'Oscar Micheaux, à l'instar de Veiled Aristocrats (1932), reposent sur cette dualité constitutive de la condition afro-américaine (voir Green 1993: 26-48).

\section{ci-contre}

fig. 2

Close Up, vol. VIII, n¹, 1931. Collection particulière, D.R. métrage expérimental muet, Borderline, tourné sur la Riviera vaudoise avec Bryher et H.D. (qui jouent dans le film), et mettant en scène Robeson et sa femme Eslanda. Cependant, le point de vue «progressiste» de Macpherson, tant dans Borderline que dans son article programmatique pour l'anthologie de Cunard, ne se déprend pas d'un fantasme primitiviste qui hante l'ensemble des mouvements modernistes de l'entre-deux-guerres ${ }^{9}$, les Noirs y étant investis comme les représentants d'une nouvelle forme de pensée archaïque par l'image qui privilégie le corps et rompt avec les carcans de la logique rationnelle.

L'ambiguïté de l'article que Macpherson écrit pour l'anthologie de Cunard est indissociable de son travail de mise en scène du couple Robeson dans Borderline. Il est ainsi possible de soutenir que l'anthologie de Cunard, sur le plan de la culture cinématographique, privilégie le point de vue inextricablement moderniste et primitiviste des Blancs - la «perspective authentique de race", dont Macpherson se fait néanmoins l'écho, s'exprimant à travers des productions cent pour cent noires destinées à un public afroaméricain, à l'instar des films d'Oscar Micheaux qui portent explicitement sur la «double conscience» et la «dualité» dans la diaspora afro-américaine telles que les a décrites W.E.B. Du Bois ${ }^{10}$.

Micheaux est considéré comme le premier réalisateur-producteur de films afro-américains réalisés en toute indépendance vis-à-vis de l'industrie du cinéma (McGilligan 2007): il a tourné, entre 1919 et 1948, vingt et un films muets et dix-huit films parlants (Green 2004: 247-248) qui s'adressent à la classe moyenne afro-américaine, mais dont la réception critique est pour le moins divisée. Résolument hybrides, ceux-ci empruntent leurs conventions aussi bien à la tradition du minstrel show qu'à des pièces de répertoire, tout en détournant les stéréotypes du cinéma hollywoodien. Alternant différents sujets d'énonciation et centres de focalisation, ces films à la trame narrative lacunaire privilégient les sujets controversés (tels que les problèmes sociaux, la rupture entre les valeurs rurales et urbaines, la corruption du clergé et le système de castes lié à la couleur de peau au sein de la société afro-américaine), tout en multipliant les regards caméra, les faux raccords et, à partir de 1930, les numéros d'attractions chantés et dansés.

De Borderline (1930, Suisse, Pool Production) de Macpherson et Body and Soul (1925, États-Unis, Micheaux Pictures Corporation) de Micheaux - les deux premiers films dans lesquels joue Robeson - se dégagent deux représentations distinctes, voire divergentes, de la question raciale. Ces longs-métrages, qui ont connu un accueil critique mitigé à leur sortie, mettent en scène les Noirs à travers une dynamique complexe impliquant plusieurs niveaux de lecture: Borderline se focalise sur la représentation des différences raciales, le corps de Robeson et le couple qu'il forme à l'écran avec sa femme aimantant le regard désirant de la caméra; Body and Soul se concentre sur la vie d'une communauté afro-américaine et blâme la logique d'exploitation du clergé. Ces deux films, il faut le souligner, constituent des exceptions dans la représentation des différences raciales: ils se situent aux antipodes des stéréotypes dégradants qui ont cours à Hollywood à l'époque, les Noirs étant le plus souvent interprétés par des acteurs blancs grimés en Noirs et réduits aux rôles de l'Oncle Tom, du Zip 


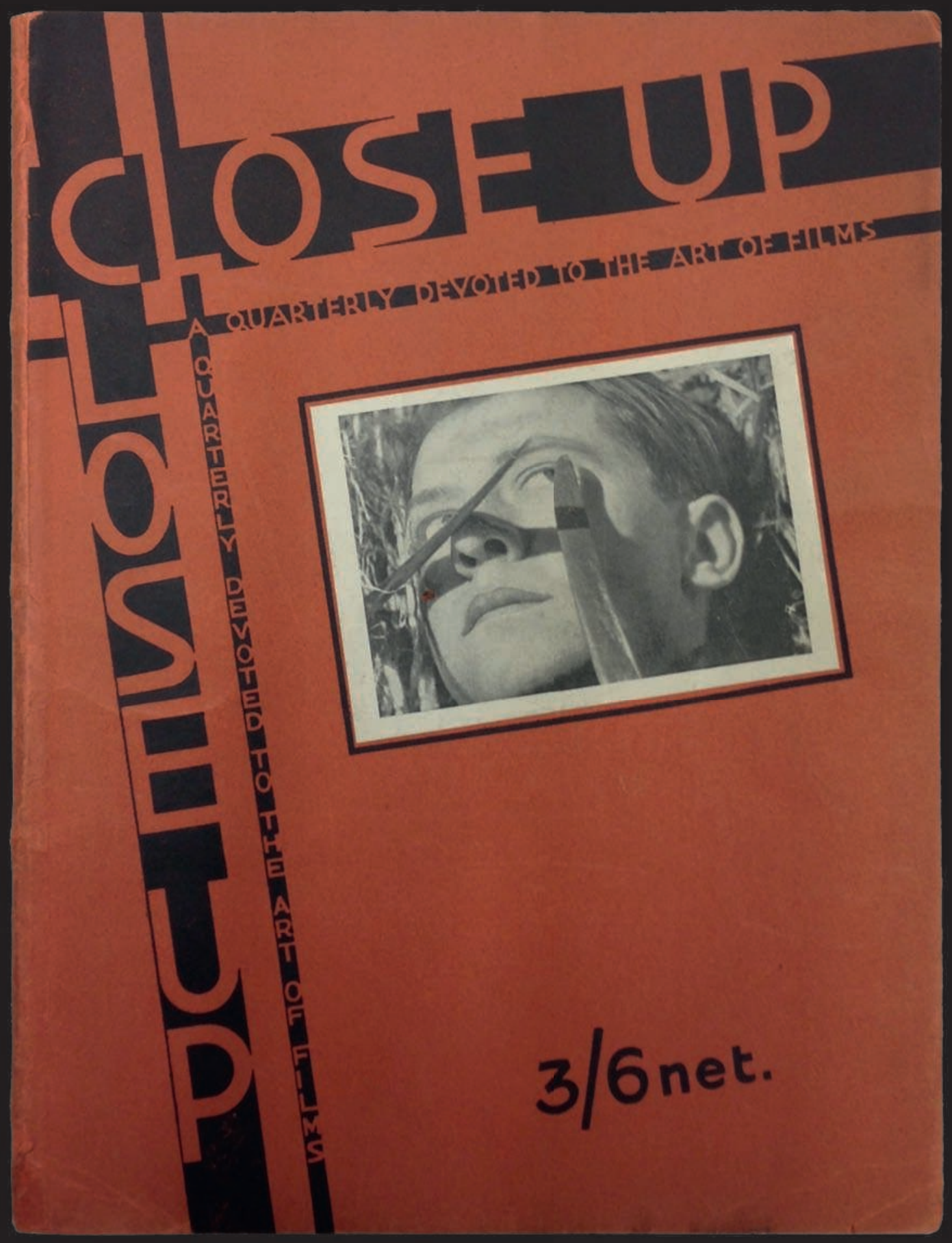




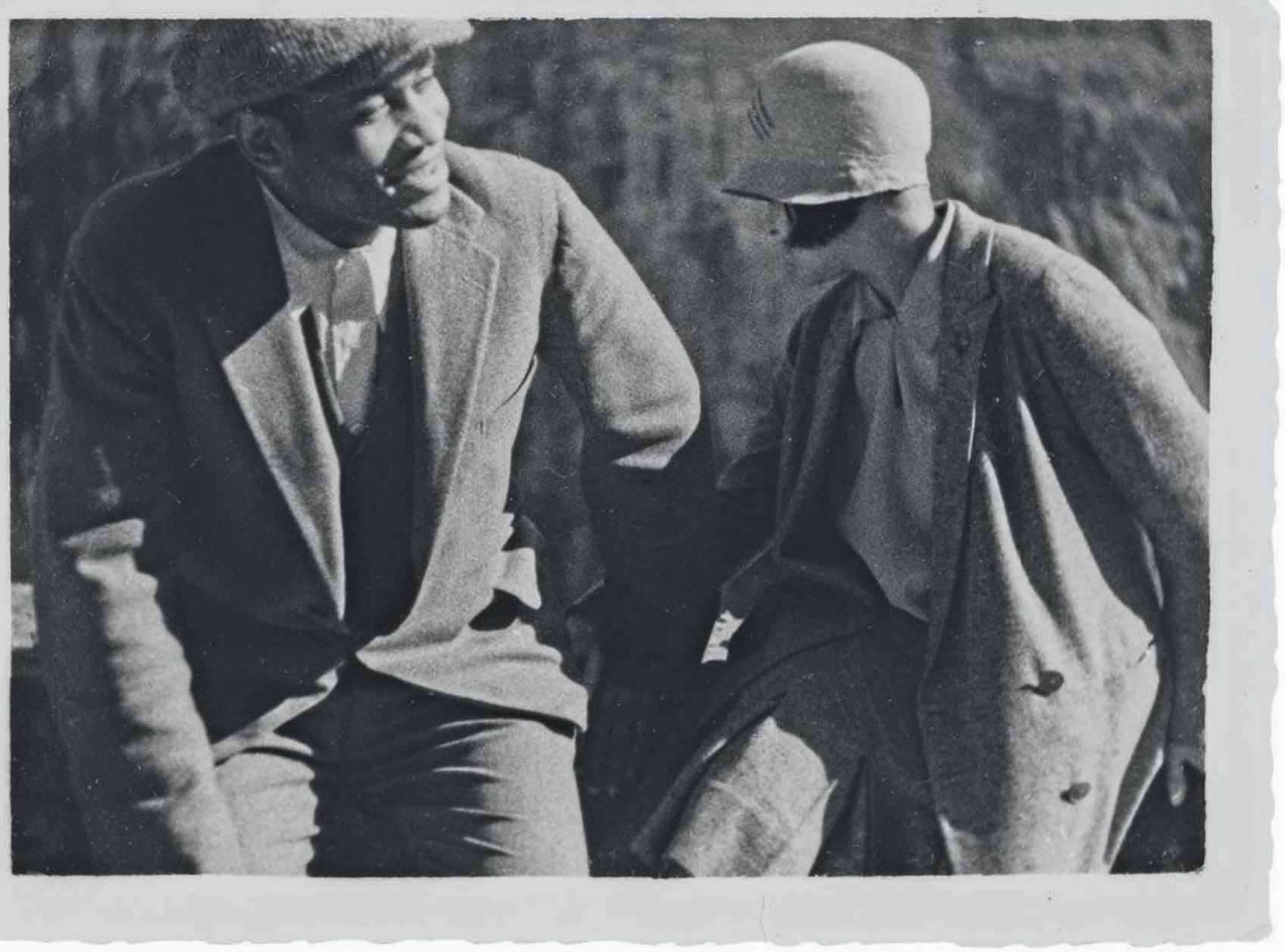

fig: 3

Paul et Eslanda Robeson

photographiés sur le tournage

du film Borderline, 1929.

Yale Collection of American

Literature, Yale University

Beinecke Rare Books

\& Manuscript Library,

GEN MSS 97. 
Coon, de la mulâtre, de la mammy et du Black Buck, pour reprendre le titre de l'ouvrage de référence de Donald Bogle ${ }^{11}$.

\section{Vers un "cinéma de race"}

Avant de retracer la logique argumentative de l'article de Macpherson, deux remarques s'imposent. D'une part, le texte paraît dans la section «Negro stars» de l'anthologie de Cunard. II n'y a donc pas d'entrée spécifique dévolue au cinéma dans le sommaire; celui-ci est mis sur le même plan que les stars ou vedettes de la musique, du théâtre, de la danse ou encore de la boxe. C'est donc bien la représentation du Noir véhiculée à l'écran qui importe, et non la mise en scène du film ou la direction d'acteurs. D'autre part, l'article se caractérise par sa dimension fortement intertextuelle: Macpherson cite longuement - sans en donner les références - son éditorial paru dans le numéro de Close Up consacré au Noir à l'écran et fait brièvement allusion à l'article de Herring paru dans le même numéro (Macpherson 1929; Herring 1929). Tout porte à croire que Cunard a sollicité Macpherson après avoir lu le dossier thématique de Close Up, ce dernier faisant à cette occasion appel à plusieurs contributeurs extérieurs à la revue et actifs sur la scène de Harlem, à savoir Geraldyn Dismond, Walter White (que l'on retrouve dans l'anthologie Negro) ou encore Elmer Anderson Carter ${ }^{\mathbf{1 2}}$. Macpherson est présenté dans l'anthologie de Cunard comme l'éditeur de Close Up, tandis que les illustrations de son article sont explicitement extraites de cette revue (en l'occurrence, un photogramme de Hearts in Dixie, avec Stepin Fetchit, et un autre de Hallelujah!, avec Nina Mae McKinney et Daniel Haynes ${ }^{13}$ ). Ces illustrations renforcent l'ambiguïté du propos de Macpherson: tirées de productions hollywoodiennes, elles ne sont pas dénuées de connotations archaïques et pittoresques, en particulier le photogramme de Hearts in Dixie, Fetchit jouant du banjo allongé paresseusement sur un tronc d'arbre.

Macpherson ouvre son article sur un mode conditionnel (la première phrase est présentée comme une pure fantaisie: «On se prend à rêver»), appelant de ses vœux l'avènement d'un cinéma authentiquement noir: "Le Nègre tenant lui-même sa propre chronique filmique, présentant luimême sa voix à l'écran, le Nègre s'exprimant intégralement et par ses propres moyens, comme les Soviétiques l'ont fait avant lui; le Nègre historiquement et socialement conscient, en pleine possession de ses moyens; le Nègre libre de dire ce que bon lui semble. » (Macpherson 1934: 335, notre traduction)

Il prolonge ainsi le titre qu'il a donné à son article: «Une Union du cinéma nègre - pourquoi pas? ", allusion à l'URSS et à son cinéma aussi bien qu'à une auto expression de race; ce programme est néanmoins grevé d'un point d'interrogation. La principale réticence de Macpherson à l'égard d'un «cinéma réalisé par des Nègres avec une équipe nègre » repose sur la crainte d'une représentation falsifiée des Africains-Américains qui équivaudrait à une opération systématique de dénigrement de la part des Blancs. C'est ce qui se passe selon Macpherson dans les magazines américains, où le Noir est présenté avec «un charme relatif, du talent et beaucoup de virilité», mais selon un point de vue «dénigrant, déformé et biaisé qui conduit à la dissimulation de la vérité» (ibid.: 336 , notre traduction). Macpherson,
11. Bogle 1989; voir aussi Cripps 1977. Le stéréotype de l'Oncle Tom repose sur un personnage vieux, dévirilisé et soumis aveuglément à l'autorité de son maître blanc: c'est l'éternel esclave noir patient et tolérant, issu du roman à succès de Harriet Beecher Stowe qui est régulièrement adapté au cinéma. Le personnage de Zip Coon est le stéréotype du Noir stupide, lent d'esprit et à la démarche nonchalante, qui cherche à s'élever au-dessus de son statut social: issu d'un milieu urbain, c'est un personnage au centre des spectacles de minstrel et popularisé au cinéma par l'acteur Stepin Fetchit. La mulâtre, dont la peau est claire et la diction aisée, est un personnage qui cherche à se faire passer pour blanc mais qui finit irrémédiablement par être démasqué, rétablissant ainsi l'interdit de la mixité raciale. Le personnage de la mammy est une nourrice, grosse et vêtue de haillons ou d'un tablier de cuisine, qui s'occupe des enfants blancs avec jovialité et servilité. Enfin, le Black Buck est un personnage libidineux, brutal, à la sexualité exacerbée et animale: c'est la figure du violeur - à la peau très foncée et au physique élancé, irrémédiablement attiré par les jeunes femmes blanches et innocentes qui hante les productions hollywoodiennes.

12. Geraldyn Dismond est une écrivaine afro-américaine et journaliste populaire qui rend régulièrement compte des activités culturelles de la scène de Harlem (notamment dans les colonnes de The Pittsburgh Courier et The Inter-State Tattler). Multipliant les réceptions (on l'appelle l'hôtesse de Harlem), elle anime également l'émission radiophonique The Negro Achievement Hour sur WABC en 1928-1929. Walter Francis White, romancier afroaméricain, est le directeur de la NAACP de 1931 à 1955 personnage incontournable de la scène de Harlem, il mène campagne contre le lynchage (notamment lors du scandale judiciaire de Scottsboro) et la ségrégation. Elmer Anderson Carter. écrivain et fondateur en 1928 de Opportunity: Journal of Negro Life, milite en faveur de la reconnaissance des droits civiques des Afro-Américains.

13. Le photogramme de Hearts in Dixie (Paul Sloane, 1929, États-Unis, Fox) est paru dans le dossier de Close Up sur les Noirs à l'écran; Dorothy Richardson consacre un article à ce film (Richardson 1929). Hearts in Dixie participe à la vogue hollywoodienne des films musicaux qui mettent en scène des acteurs noirs au moment de la généralisation du sonore, alternant à travers une structure en épisodes les chants et les danses autour d'un personnage qui reconduit le stéréotype de I'Oncle Tom. Le photogramme de Hallelujah! (King Vidor, 1929, États-Unis, MetroGoldwyn-Meyer) a été publié dans le numéro de mars 1930 (Close Up V [3]) ; d'autres photogrammes de ce film ont été reproduits dans le dossier consacré à la censure (février 1929 IV [2]), tandis que Herring commente brièvement la production hollywoodienne de King Vidor (Herring 1929 : 101). Hallelujah! reconduit les stéréotypes de la mulâtre tragique et du Black Buck qui se déguise en prédicateur (artifice qui permet à Vidor de multiplier les scènes de chants traditionnels), mais inverse leurs connotations négatives en humanisant les personnages. Premiers films sonores produits à Hollywood avec une équipe d'acteurs exclusivement noirs, Hearts in Dixie et Hallelujah! sont généralement investis par la critique de l'époque comme des films progressistes quant à la représentation des races, malgré leur stéréotypie et l'exploitation du folklore afro-américain. 


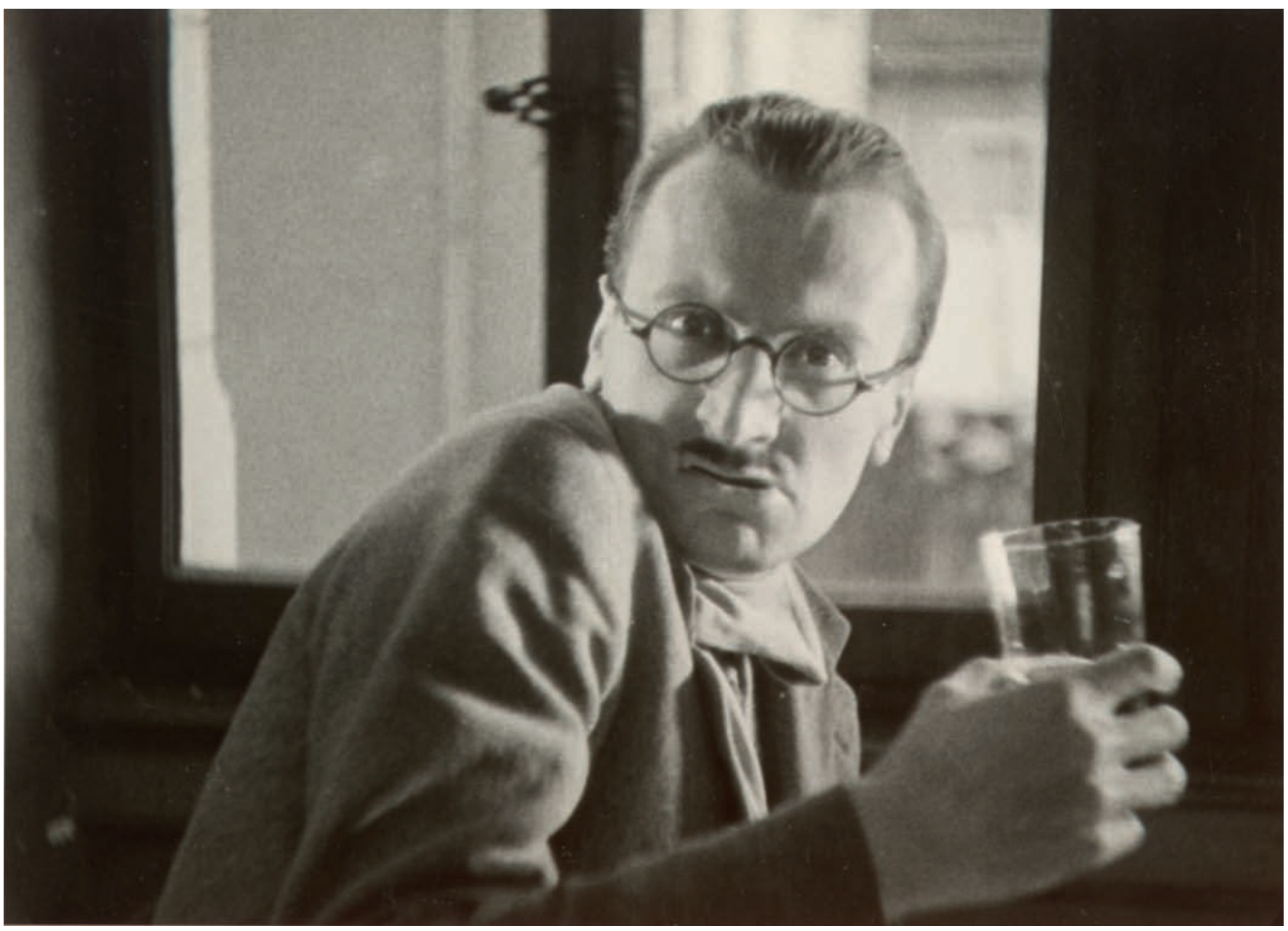

fig. 4

Kenneth MacPherson

photographié sur le tournage

de Borderline, 1929. Yale

Collection of American

Literature, Yale University

Beinecke Rare Books

\& Manuscript Library,

GEN MSS 97.

\section{ci-contre}

fig. 5

Photogrammes de Paul

Robeson dans Body and Soul

(1925) d'Oscar Micheaux,

1925. D.R. 


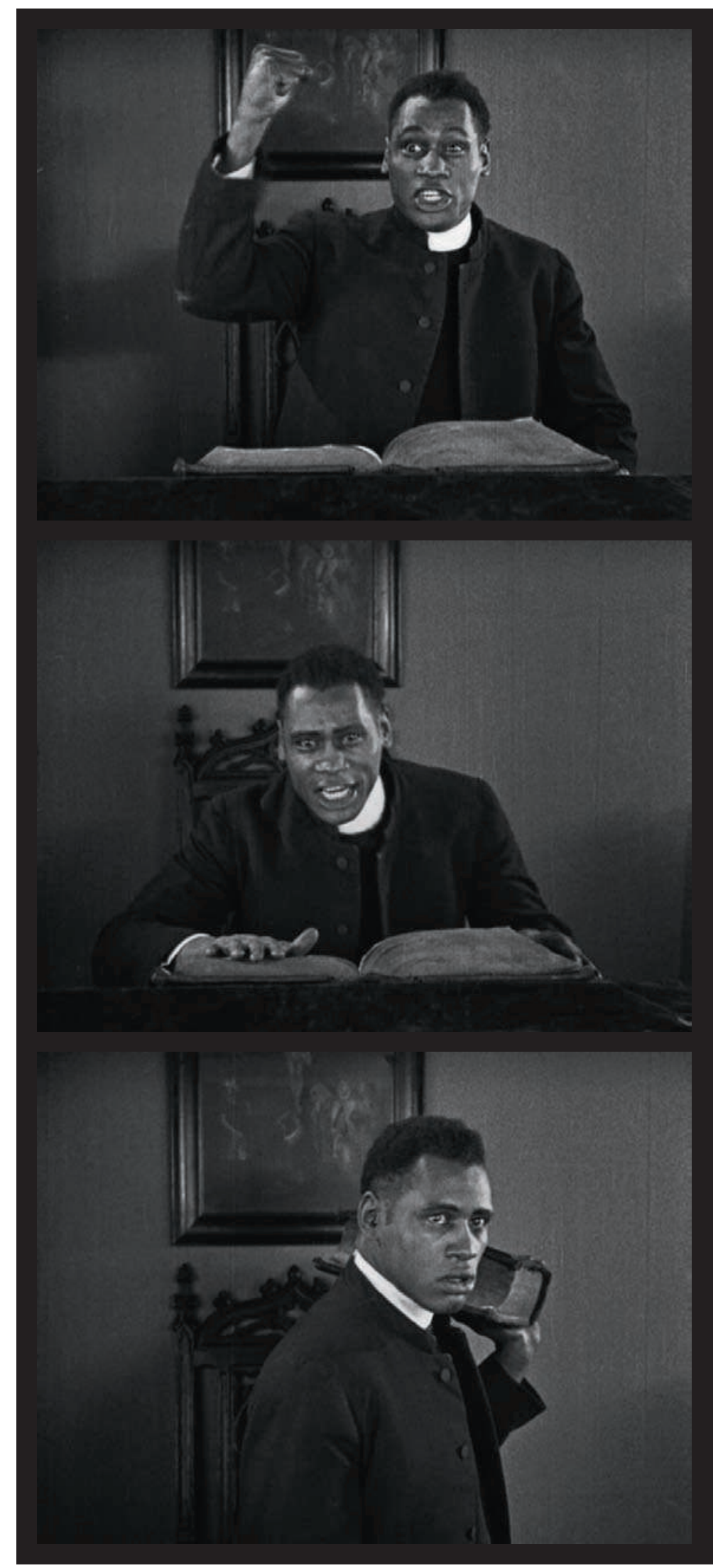


14. Macpherson écrit notamment: "Il y a plus que l'ombre d'une promesse dans la jungle, dans cet élancement agile qui affirme soudainement quelque chose d'irréfutable, en face de ce qui nous frappe comme pure beauté. [...] II agite d'amples mains de race qui, comme la vie, touchent tout ce que le monde contient. » (Macpherson 1929: 87-88 [notre traduction]

15. Voir Cunard 1933 274-278. Nancy Cunard revient plus longuement sur ce scandale judiciaire dans son anthologie ( Scottsboro - and other Scottsboros ", in Cunard (éd.) 1934 : 243-268).

16. La NAACP ne manque pas de souligner les liens entre le livre de Dixon et l'adaptation de Griffith. W.E.B. Du Bois peut ainsi écrire dans The Crisis :

"Récemment, ce livre vicieux [The Clansman] a été adapté au cinéma. Avec une grande habileté, la reconstitution du livre est précédée par de nombreuses images de guerre étonnamment bonnes; ensuite, dans la seconde partie, The Clansman est mis en scène en représentant le Nègre comme un fou ignorant, un violeur vicieux, un politicien vénal et peu scrupuleux ou un idiot fidèle mais débile. II n'est pas étonnant qu'un homme [Dixon] capable de falsifier aussi grossièrement l'histoire n'ait jamais été en mesure de produire une œuvre littéraire qui puisse attirer l'attention, si l'on excepte les cas où il tire parti des antagonismes brûlants de race. » (W.E.B. Du Bois, "The Clansman », The Crisis, mai 1915, p. 33 in Everett 2001: 102-103, notre traduction) en citant l'éditorial qu'il a écrit pour le numéro de Close Up consacré aux Noirs à l'écran, décrit Fetchit dans Hearts in Dixie (en élidant cette fois le nom de l'acteur et le titre du film) comme l'incarnation d'une pure beauté animale, correspondant à un fantasme primitiviste ${ }^{\mathbf{1 4}}$. Reprenant ses propos dans Close Up, il précise sa pensée en assimilant le personnage de Fetchit à l'expression du «sens de la virilité ou de la solidarité » qui définit selon lui la «négritude». II rapporte alors une discussion avec Robeson, probablement à l'été 1929, avant qu'il le mette en scène dans Borderline: Robeson craint que la représentation de l'Afro-Américain à l'écran, du moins dans les productions hollywoodiennes, ne se réduise à l'imagerie pittoresque de la "cueillette de coton»; Macpherson recherche au contraire un moyen de mettre en scène et de dénoncer la ségrégation et le lynchage des Noirs à travers l'image de «fermiers fous, avides de lynchage, et de leurs victimes" (Macpherson 1934: 336, notre traduction). Revenant sur le temps présent et son article pour l'anthologie de Cunard, il reconnaît que, dans une production telle que Hallelujah!, la ségrégation n'est pas abordée; et il préfère pour sa part prendre pour modèle Georgia Nigger de John Spivak (Spivak 1932) qui n'a pas encore été publié au moment de sa discussion avec Robeson mais constitue son idéal à atteindre à l'écran. Fustigeant le système pénal de la Géorgie, le journaliste militant y décrit avec précision les conditions de travail et les mauvais traitements des prisonniers afro-américains dans un État du sud des États-Unis; malgré le masque de la fiction, Spivak s'appuie sur des documents officiels bien réels (procès-verbaux de coups de fouet, documentation des tortures infligées aux bagnards enchaînés, etc.).

Est-ce à dire que Macpherson envisage Borderline comme un film pamphlétaire exposant les mécanismes qui conduisent au lynchage en ayant à l'esprit le scandale de Scottsboro, dénoncé par Cunard notamment dans les pages de Close Up en $1933^{15}$ ? Difficile de trancher sur le plan des intentions de Macpherson; mais, au visionnage de Borderline, la radicalité de sa démarche peut être relativisée. Le film participe néanmoins à une volonté d'inversion de la logique raciste des productions hollywoodiennes, notamment et notoirement celle de The Birth of a Nation (D.W. Griffith, États-Unis, David W. Griffith Corp., 1915), adaptation à l'écran de The Clansman: An Historical Romance of the Ku Klux Klan de Thomas Dixon. On le sait, The Birth of a Nation a donné lieu à des protestations et des émeutes dans plusieurs villes des États-Unis - la campagne d'interdiction du film menée par la NAACP n'aboutissant pas ${ }^{\mathbf{1 6}}$. Pour répondre à la superproduction de Griffith, qui a participé à la renaissance du Ku Klux Klan, la Booker T. Washington Organization du Tuskegee Institute conçoit le projet d'un film panafricaniste, The Birth of a Race. Longtemps avorté, The Birth of a Race a finalement été tourné par John Noble sous une forme émoussée en 1919 (Green 2004: 21-22). Le contrat est en revanche pleinement rempli avec Within Our Gates (1920, États-Unis, Micheaux Book and Film Company), film radical où Micheaux renverse littéralement le scénario de Griffith en montrant le lynchage de Noirs innocents, les mécanismes du péonage et la répression menée par les milices. Son titre détourne l'épigraphe de A Romance of Happy Valley de Griffith: "Ne fais pas de mal à l'étranger qui est dans tes murs, de peur que ce ne soit toi qui sois blessé [Harm not the stranger/Within your gates/Lest you yourself be hurt]. " Le film s'est heurté à la censure car il se fait le porte-parole de la cause afro-américaine (le 
dernier flash-back met en scène le lynchage d'un Noir et le viol évité de justesse de la jeune héroïne mulâtre par un riche propriétaire terrien blanc son père d'adoption). Les publicités annonçant la sortie de Within Our Gates - dans une version tronquée suite aux interventions de la commission de censure - le présentent comme «la prédication la plus importante contre les préjugés de race» (Gaines 2001: 68, notre traduction).

Le projet de film de Macpherson - qui ne se confond pas avec Borderline - répond à une telle entreprise de destitution et de renversement du scénario de Griffith. II est cependant peu probable que Macpherson ait eu l'occasion de voir Within Our Gates, le film circulant exclusivement dans le réseau des salles afro-américaines; en revanche, il connaît les films de Micheaux par ouï-dire (Dismond renvoie dans le dossier de Close Up consacré à la représentation des Noirs à l'écran à la Micheaux Pictures Company [Dismond 1929: 96]). Par ailleurs, Macpherson reste attentif à la carrière de Robeson à l'écran, publiant en décembre 1933 un article de Herman G. Weinberg consacré à The Emperor Jones de Dudley Murphy (une adaptation de la pièce éponyme d'Eugene O'Neill qui a participé au succès de Robeson en tant qu'acteur de théâtre en 1924 [Weinberg 1933]).

Quoi qu'il en soit, Robeson, à l'occasion de cette discussion avec Macpherson, rejette sans concession les productions hollywoodiennes mettant en scène des Noirs, telles celles qui illustrent l'article de ce dernier dans l'anthologie de Cunard. En septembre 1933, il réitère sa critique dans Film Weekly:

L'insistance du box-office à toujours représenter le Nègre sous les traits d'un clown a dénaturé les deux films nègres qui ont été réalisés à Hollywood, Hallelujah! et Hearts in Dixie. Dans Hallelujah!, ils se sont emparés du Nègre et du culte religieux pour les ridiculiser. [...] Hollywood ne peut s'imaginer le Nègre qu'à travers l'imagerie de la plantation, [...] le Nègre du «Poor Old Joe » et de la «Swanee Ribber ». Il est absurde de recourir à ce mode d'expression pour représenter le Nègre moderne, aussi absurde qu'il le serait de présenter l'Angleterre moderne dans les termes d'une ballade élisabéthaine ${ }^{\mathbf{1 7}}$.

Macpherson, dans son article publié dans l'anthologie, oppose à cette représentation stéréotypée, ainsi qu'à la vogue des «courts films entièrement chantants de la Metro-Goldwyn-Mayer» mettant en scène des Noirs - autre cible de Robeson -, une forme de cinéma pamphlétaire «dont l'impact est primitif, physique, cruel et insoutenable» (Macpherson 1934: 336, notre traduction). Son hypothèse est qu'un tel film serait censuré et son réalisateur accusé d'être un «dangereux bolchévique». II parle ici explicitement de son propre projet de film, auquel pourrait se rallier un militant communiste comme Robeson mais en aucun cas Micheaux, partisan de l'assimilation et admirateur de Booker T. Washington (dont le programme politique repose sur l'éducation et le développement économique de la classe moyenne afro-américaine, qui conduirait à l'égalité sociale et aux droits civiques sans remettre fondamentalement en cause les mécanismes de la ségrégation). Macpherson cite ici à nouveau son éditorial de Close Up: «l'homme blanc "confronté à
17. Entretien de Doris Mackie avec Paul Robeson, "Negro films I want to make ", Film Weekly, $1^{\text {er }}$ septembre 1933 , cité par Willis 1998: 76 (notre traduction). Robeson maintiendra cette position par la suite : voir notamment Paul Robeson, "Hollywood's "Old plantation tradition" is "offensive to my people" ", The New York Times,

24 septembre 1942, in Foner (éd.) 1978: 142. 
18. La citation non tronquée souligne une représentation inauthentique de la race: "Confronté à une instabilité (la sienne) qu'il qualifie de "problème de race", I'homme blanc, aussi bienveillant soit-il, dresse toujours le portrait du Nègre comme cela l'arrange. La bienveillance constitue en fait le principal danger. " (Macpherson 1929: 87, notre traduction)

19. Dans les années 1920 , Lev Koulechov dirige en URSS un atelier où il expérimente de manière collective le jeu d'acteur (la précision dans les gestes et la performance physique caractérisant le "modèle» filmique) et le montage au cinéma. Il tourne, le plus souvent sans pellicule, différents exercices avec son collectif. Voir Albera 1994 ; Koulechov 1994.

\section{ci-contre}

fig. 6

Photographie de plateau du film Borderline, 1929. Yale Collection of American Literature, Yale University Beinecke Rare Books \& Manuscript Library, GEN MSS 97. une instabilité - la sienne - qu'il qualifie de problème de race ${ }^{18}$ "! " | | prend toutefois ses distances par rapport à ce qu'il a écrit en 1929: s'il s'incluait alors lui-même dans la catégorie de l'«homme blanc" qui cherche à filmer des Noirs, ses propos servent cette fois à montrer du doigt les «imbéciles" qui dénonceraient le film militant qu'il a l'intention de tourner. Borderline, laisse-t-il donc entendre implicitement, n'emprunte finalement pas la voie du pamphlet et de la polémique.

Macpherson suspend alors sa discussion avec Robeson et son projet de film pour revenir à la question de la spécificité du médium cinématographique: le film est-il un support adapté à l'expression de la culture noire? II craint en effet que celui-ci ne favorise un glissement du «drame» vers le «mélodrame», du «fait» vers le «tract» (Macpherson 1934: 336, notre traduction). Opposant la musique et la danse - et n'hésitant pas à employer le terme d'«atavique " pour caractériser la «pulsation» dynamique de ces formes d'expression - à la littérature afro-américaine, qu'il juge trop édulcorée, il laisse la question ouverte, le cinéma constituant un médium "dynamique» qui ne se limite pas au mot et à l'écriture. Son modèle, une fois encore, est le cinéma soviétique, en particulier le travail collectif tel que le pratique Lev Koulechov dans son atelier ${ }^{19}$ : un cinéma «à la quintessence nègre" (Macpherson 1934: 336, notre traduction) ne pourra se développer qu'à travers une expérimentation commune par-delà les nations. II peut ainsi conclure en citant Lénine - «Pour nous, le cinéma est le plus important des arts!» -: le cinéma afro-américain repose sur le travail de réalisateurs et d'acteurs noirs, auxquels incombe la tâche de s'approprier ce moyen d'expression moderne. Le leitmotiv de son article, "le Nègre tenant luimême sa propre chronique filmique", indique bien quels sont pour lui les enjeux d'un cinéma de race authentique: celui-ci repose sur une chronique, une simple consignation de faits, à travers un mode d'expression rythmique et dynamique.

Sans craindre le paradoxe, Macpherson considère la constitution d'un cinéma afro-américain et la réalisation de ses propres films comme un seul et même projet. Cette double voie d'expression - l'autoreprésentation et la mise en scène stylisée des Noirs - peut être ressaisie à travers une analyse des modes de représentation mis en jeu dans Body and Soul et Borderline.

\section{Borderline, ou le fantasme archaïque du dieu noir}

II est possible d'adopter deux interprétations concurrentes de Borderline, que l'on retrouve dans la littérature secondaire sur le film ayant intégré les apports des race studies: il peut être considéré comme un film antiraciste qui démonte les mécanismes du lynchage (c'est le point de vue d'Annette Debo [Debo 2001]); dans une perspective diamétralement opposée, il peut être envisagé comme un film primitiviste, présentant le Noir comme une altérité désirable qui échappe aux contraintes de la socialisation (voir plus loin l'argumentation de Jean Walton [Walton 1998: 395416; Walton 1999: 243-270]).

Selon Debo, Borderline inverse le scénario du lynchage, dont la justification repose sur une offense à la suprématie blanche (un Noir accusé 


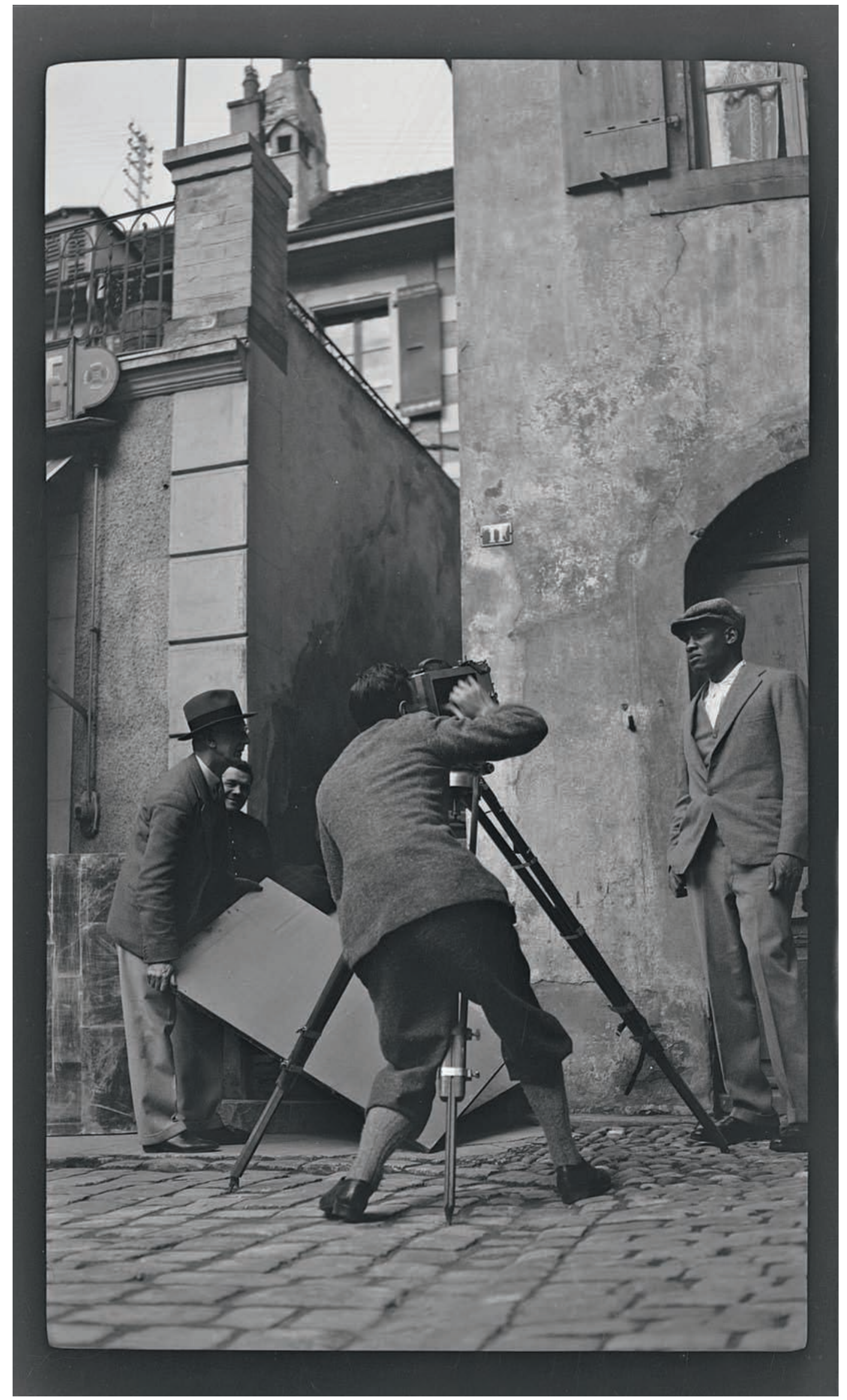


fig. 7

Paul Robeson dans

Borderline, 1929. Yale

Collection of American

Literature, Yale University

Beinecke Rare Books

\& Manuscript Library,

GEN MSS 97.

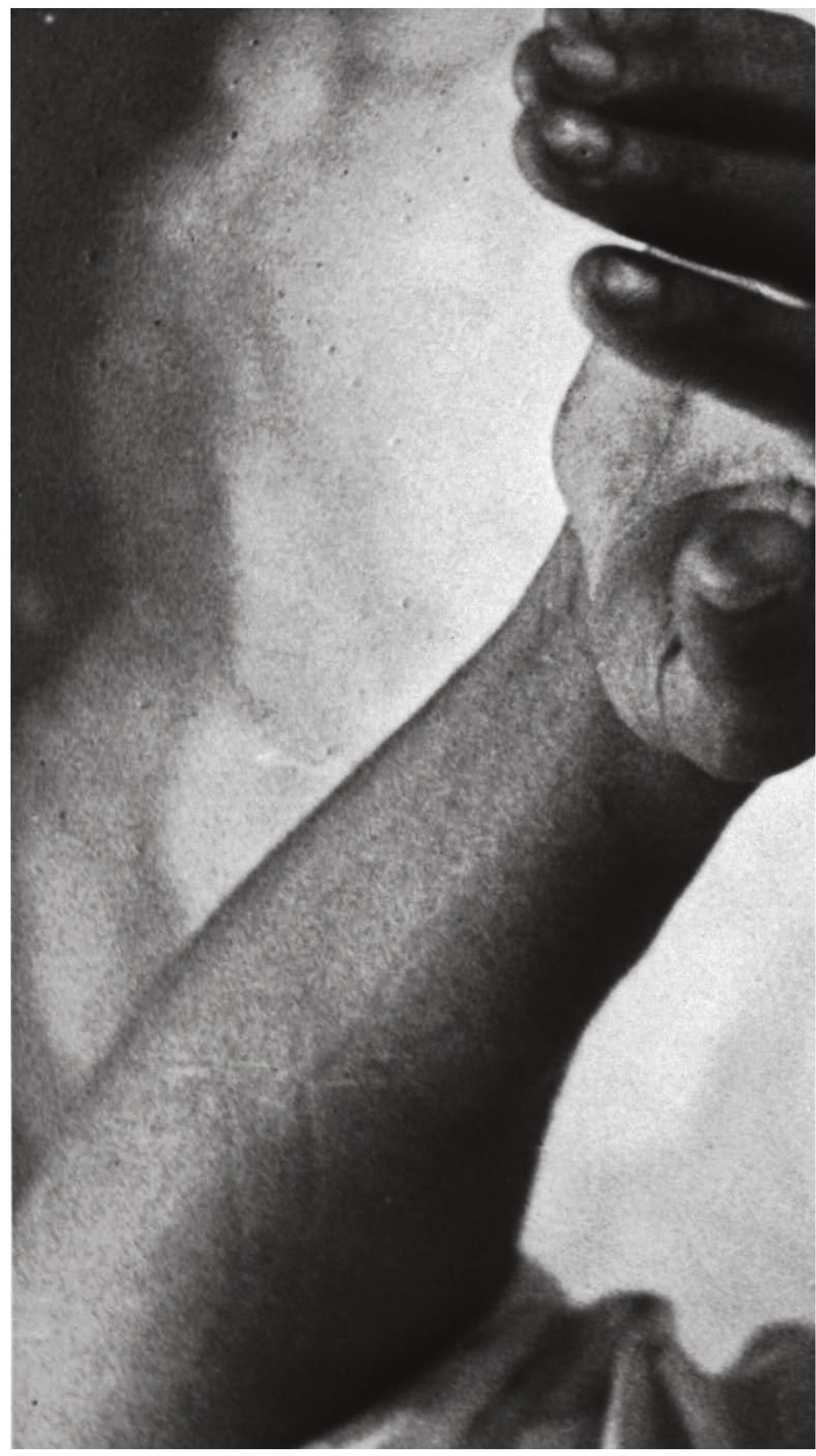




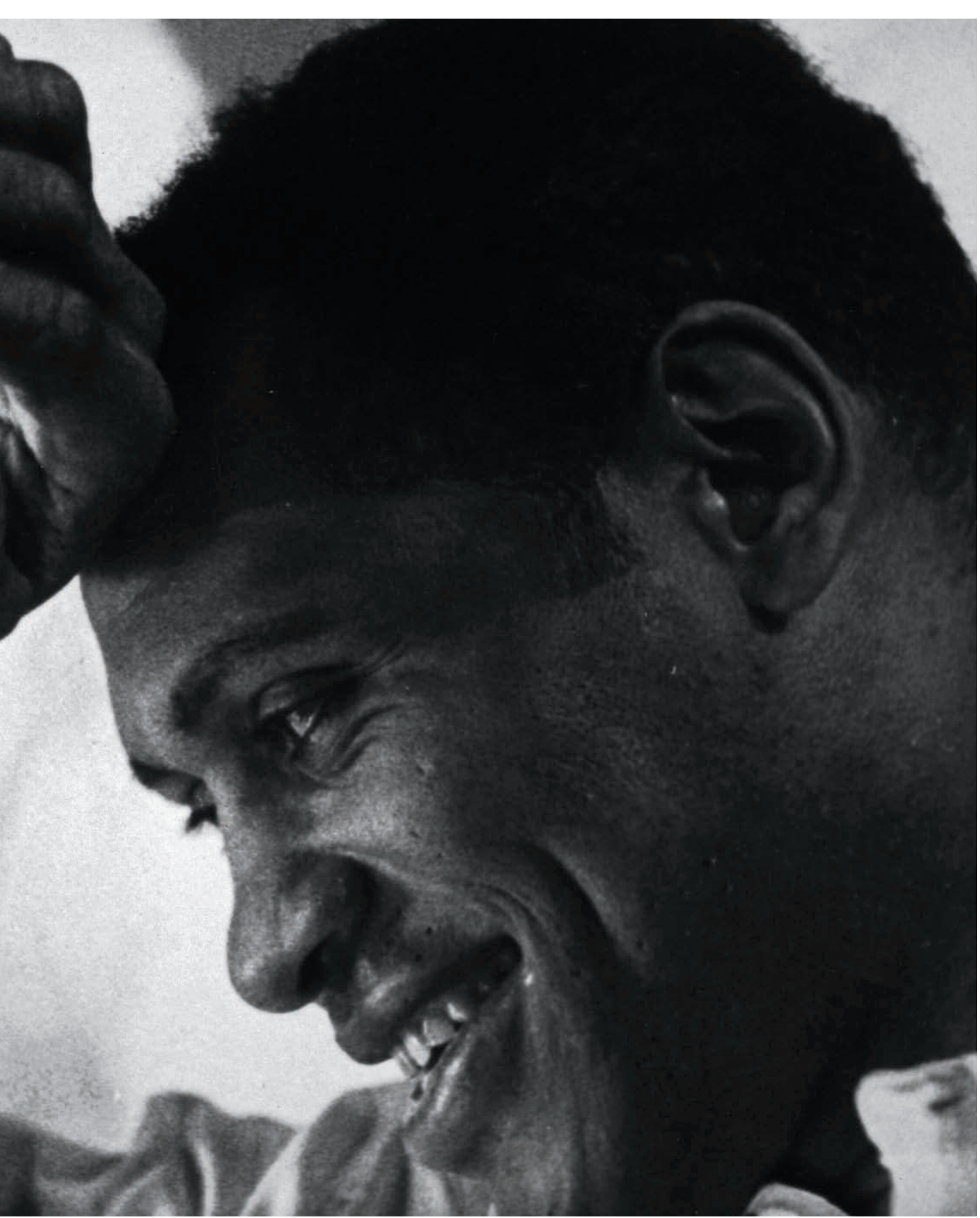


20. Journal d'Eslanda Robeson, cité par Duberman 1988: 131 (notre traduction).

21. "La première expérience artistique de l'humanité, comme nous le savons tous, fut la fameuse écriture "pictographique". [...] nous commencerons peut-être à "écrire" nos romans et nos pièces au moyen de "pictogrammes". " (H.D. 2006: 29)

22. Paul Robeson, "Primitives", The New Statesman and Nation 8 août 1936, in Foner (éd.) 1978: 109-113. du viol d'une femme blanche). Dans le film, en effet, un homme blanc (joué par Gavin Arthur) entretient une liaison avec une mulâtre (Eslanda Robeson) et tue sa propre femme (interprétée par H.D.); il est néanmoins acquitté par le tribunal. L'homme noir (Paul Robeson), qui s'est rendu dans ce village pour retrouver sa femme, la mulâtre, est expulsé, la communauté maintenant ainsi «son hégémonie blanche» (Debo 2001: 372 , notre traduction). Debo accorde une fonction centrale à la scène de conflit qui se déroule dans un café, au terme de laquelle Robeson assomme le personnage de notable joué par Macpherson (à propos de cette scène étrangement teintée de masochisme, Macpherson écrit aux Robeson: «Je suis incroyablement écœurant dans le rôle de l'homme que Pete met à terre! » [Ibid.: 375, notre traduction]). Un montage très court fait alors allusion à la pendaison d'un Noir par une foule déchaînée: des flammes, des poings brandis et une affiche représentant un corps noir sont superposés. Si l'on radica-lise la logique de cette lecture, quitte à forcer l'argumentation de Debo, cette plaidoirie contre le racisme s'apparente à une variation autour de Within Our Gates: le personnage d'Eslanda Robeson, mulâtre qui fait le lien entre le monde blanc et le «nouveau Nègre», occupe la position de victime, à l'instar de l'héroïne du film de Micheaux; I'homme blanc joué par Arthur, infidèle et meurtrier de sa femme, n'est jamais inquiété, tout comme le riche propriétaire blanc de Within Our Gates. Dans tous les cas, et comme le laisse entendre Macpherson dans l'anthologie de Cunard, indépendamment de ce jeu de parallélismes et de correspondances, Borderline inverse les prémisses raciales de The Birth of a Nation.

Le récit qu'Eslanda Robeson consigne dans son journal devrait cependant nous inciter à relativiser le caractère progressiste de Borderline:

Kenneth [Macpherson] et H.D. avaient pris l'habitude de nous faire rire aux larmes avec leurs idées naïves sur les Noirs, au point que Paul et moi devions parfois refaire tout notre maquillage. Avec eux, nous n'avons jamais ressenti une seconde que nous étions des gens de couleur ${ }^{20}$.

Eslanda Robeson souligne la naïveté et les représentations stéréotypées des réalisateurs, Borderline reposant sur l'opposition entre nature et culture, primitivisme et désordre mental, monde noir et civilisation blanche. La spécialiste de littérature américaine et de cinéma Jean Walton, qui confronte les outils de l'analyse psychanalytique aux études de race et de genre, a identifié le principal intertexte de Borderline, systématisant l'image de l'homme primitif et naturel: un essai de Sigmund Freud de 1908 intitulé "La morale sexuelle "civilisée" et la maladie nerveuse des temps modernes» (Freud 1969: 28-46). Le couple blanc est présenté comme instable, névrotique, dégénéré, tandis que le couple noir incarne le mythe rousseauiste du «bon sauvage». Cette fascination pour le primitivisme - fantasme exclusivement blanc qui ressurgit de façon récurrente dans le modernisme - se retrouve dans l'essai que H.D. a écrit sur Borderline en 1930: pour elle, le film de Macpherson constitue l'actualisation la plus parfaite d'une «écriture pictographique" ("picture" writing 21), c'est-à-dire la formulation d'une pensée primitive par l'image. Notons que ce mythe est également présent dans certaines déclarations de Robeson ${ }^{\mathbf{2 2}}$, celui-ci 
opposant l'expression par «symboles concrets» à la pensée en «concepts abstraits». Le film est traversé par la polarité Noir/Blanc suivant une logique d'inversion des valeurs axiomatiques attachées aux représentations de race (les Blancs étant connotés négativement). H.D. peut ainsi écrire:

Ces personnages sont eux-mêmes les énigmes, ils demandent «pourquoi» et ils demandent «comment» [...]. Quand un Africain n'est-il pas un Africain? Alors qu'à l'évidence il est un dieu de la terre. Quand une femme n'est-elle pas une femme? Alors qu'à l'évidence elle est froide et glacée, une mouette empaillée (H.D. 2006: 7-8).

Symboles incarnés, les personnages sont envisagés comme des pictogrammes composant un système d'oppositions et de polarités (Paul Robeson en tant que «dieu de la terre»; H.D., « une mouette empaillée»):

[Pete, le personnage de Paul Robeson] est stabilisé, ferme, la terre. [...] Le géant noir est les hauts nuages, le cumulus blanc concentré dans des cieux plus hauts. Ses compagnons masculins blancs, à l'inverse, sont les ombres du blanc, sombres, névrosés. La tempête gronde; une malédiction biblique prédit que «ceux qui vivent de l'épée périront par l'épée». Ou, comme c'est le cas ici, «ceux qui ont vécu dans le refoulement érotico-névrotique en périront ». (Ibid.: 11)

Le Noir et les Blancs campent la dissymétrie entre un désir éthéré (les «hauts nuages» dans des «cieux plus hauts») et une pulsion de mort (les «ombres du blanc, sombres»), qui se traduit dans le film par l'opposition entre la figure heureuse du couple noir et la dislocation de la communauté soi-disant civilisée (par ailleurs, la transposition à l'écran d'expressions lexicalisées - la tempête gronde, ceux qui vivent de l'épée périront par l'épée - et de jeux de mots constitue l'une des singularités de Borderline, sur laquelle l'on ne s'arrêtera pas ici ${ }^{23}$ ). H.D., qui a joué un rôle indéniable dans l'écriture du scénario et le montage du film, décrit la logique de composition de Borderline comme un processus de mise en relation de motifs qui acquièrent une fonction «abstraite». Dans des termes extrêmement chargés sur le plan des oppositions raciales, elle développe ce clivage entre le personnage de Paul Robeson et celui qu'elle interprète elle-même à l'écran:

Un petit bidon d'huile de chauffage, par exemple (abstraction moderniste concise), renvoie à l'épaule d'un géant noir. L'huile et la chaleur sont associées à un front noir, cette grande tête qui se penche en avant, véritable géant terrestre, cependant que l'air et la lumière, figurés dans le rideau que le vent gonfle, enchaînent sur l'abstraction victorienne d'une mouette morte empaillée, et de là, via les éclairs accélérés d'une séquence inéluctable, jusqu'au visage altéré d'une femme (H.D. 2006: 7).

Le bidon d'huile, la chaleur dégagée par le réchaud et le front noir de Paul Robeson, suivant l'isotopie du feu et du désir, s'opposent au rideau gonflé par le vent, à la mouette empaillée et au visage altéré de H.D. qui empruntent à la thématique du linceul, de la mort et de la déraison. Au clivage Noir/Blanc, nature/culture, s'ajoute la polarité homme/femme
23. Sur ce point, voir Bovier 2009: 243-276. 


\section{RED ROSES \\ FOR B RONZE "H.D"}

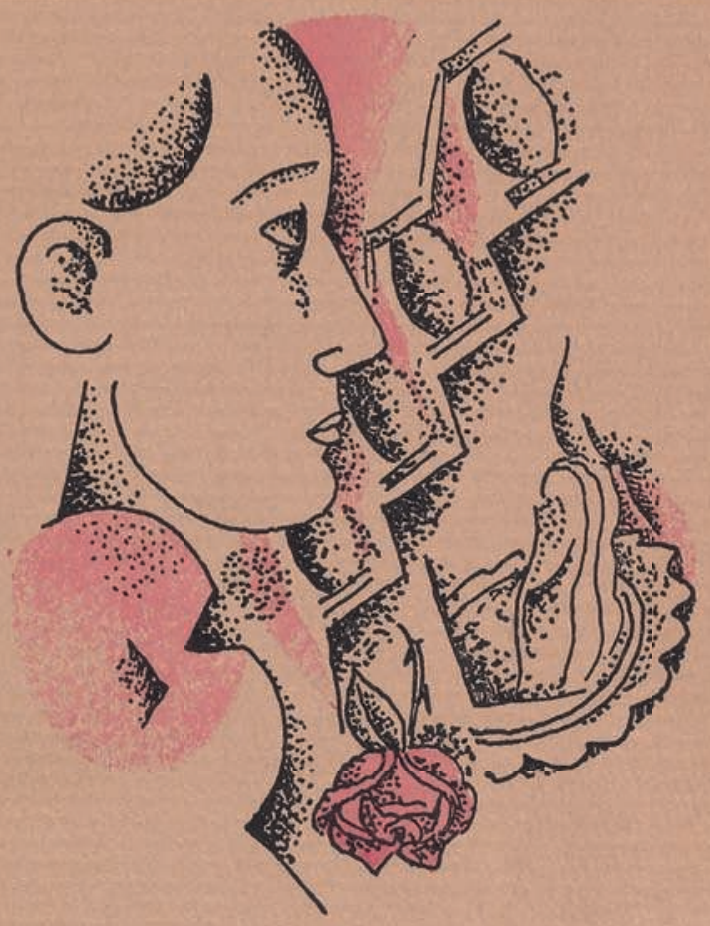

T H E Random House, New York, 1929 
suivant une inversion comparable à celle des représentations de race (le corps masculin est porté par le désir, et le corps féminin dévitalisé).

La déification du personnage de Paul Robeson se double également d'une érotisation de son corps, le plaçant dans une position d'assujettissement qui perturbe l'identité de genre et la différenciation entre les sexes. Certains mouvements de caméra parcourent son corps comme une terra incognita, sous la pression du désir. Celui-ci s'entrelace ou se superpose à une cascade grâce au montage de brèves séquences de photogrammes induisant un effet de surimpression. Parfois, c'est la mise en scène et le cadrage qui le subliment. Dans Close Up, à des fins de promotion du film, et dans The Architectural Review, à l'occasion d'une analyse de séquences, Macpherson met en relation un portrait de Robeson, dont la composition est remarquablement stylisée, avec un photogramme de H.D. gisant sur le sol, inanimée. Robeson est cadré en gros plan, souriant, une rose à l'oreille, et brandit derrière sa tête un plateau qui dessine une auréole. Macpherson légende en ces termes les images dans Close Up:

Ces deux photogrammes suggèrent les gages de la vertu et les gages du péché. En haut, Paul Robeson apprécie sa canonisation autoordonnée; en bas, Helga Doorn [pseudonyme de H.D. à l'écran] donne son interprétation de ces petites morts dont nous mourons tous. (Close Up VII (2), 1930: 130, notre traduction)

Robeson compose un tableau vivant, sur le mode du pastiche ou de la mascarade: Dieu noir et archaïque, il s'offre aux regards comme une icône à vénérer. Mais il est dans le même temps réduit à une posture passive et contrainte, ses mouvements étant restreints, presque figés. La serveuse (jouée par Charlotte Arthur, qui forme dans Borderline un couple avec la patronne de l'hôtel incarnée par Bryher) répond au geste de «travestissement» de Pete (sa canonisation auto-ordonnée) par une «mascarade» sauvage: tenant un couteau entre les dents, elle accomplit une danse frénétique, hystérique, mais somme toute inoffensive. Dans The Architectural Review, Macpherson commente cette brève séquence:

Cette scène et la séquence pendant laquelle Thorne [Gavin Arthur] tue Astrid [H.D.] sont liées. Sur l'impulsion du moment, Pete [Paul Robeson], saisissant une rose, mime un danseur espagnol. La serveuse, suivant son mouvement par jeu, s'empare d'un couteau. [...] Cette parodie de danse espagnole dans le bar de l'hôtel, avec couteau et rose, est une sorte de commentaire satirique du destin de ces personnes «sur le fil du rasoir » [borderline] qui, n'ayant pas prise sur la vie et dépassées par les événements, peuvent faire pleurer les anges et rire les dieux. (Mercurius 1930: 258, notre traduction)

Selon l'analyse de Macpherson, Robeson se retrouve ainsi dans la même position féminisée que H.D. - celle d'une victime simulant la mort avant de l'éprouver réellement. La composition du plan fétichise le personnage de Robeson, divinité noire à la sensualité exacerbée. Paradoxalement, cette glorification du corps «naturel» et «primitif» de l'acteur se double d'une iconographie qui contredit ou du moins canalise sa vitalité, à savoir la

\section{ci-contre}

fig. 8

Red Roses for Bronze,

Random House, New York, 1929. Yale Collection of American Literature, Yale University Beinecke Rare Books \& Manuscript Library, JWJ MSS 26. 
24. «Si je pouvais prendre une masse de bronze/et assouvir/mes doigts crispés/ en un travail extatique,/si je pouvais façonner/les yeux, la bouche et le menton,/ si je pouvais prendre le bronze noir/et marteler/la ligne sous ta lèvre inférieure/ (le sourire un peu moqueur/ un peu cynique/que tu as choisi d'arborer)/si je pouvais soulager mes doigts et mon esprit/avec des coups,/ des coups,/des coups,/des coups,/des coups contrequelque chose (la pierre, le marbre, une intention, stable, matérialisée)/la paix,/ même la magie du sommeil/ pourraient à nouveau venir. " (H.D. 1983: 211,

notre traduction)

25. Nickolas Muray est un photographe de mode qui a travaillé dans les années 1920 pour Harper's Bazaar, Vanity Fair, Vogue ou encore le New York Times. II a réalisé le portrait de nombreuses célébrités, notamment de stars hollywoodiennes. Certaines photographies de nus de Robeson seront publiées sous forme de cartes postales. Sur la série Nude Kneeling, voir Stewart 1998: 135-138, 143-152.

26. Dans La Question de I'analyse profane (1926), Freud décrit la «vie sexuée de la femme adulte " comme un "dark continent pour la psychologie" (Freud 1994: 36). Cet amalgame entre la sexualité féminine et le continent noir est révélateur de la construction psychanalytique de l'altérité sur un mode négatif : c'està-dire non masculin et non occidental.

\section{ci-contre}

fig. 9

Nickolas Muray, Nude Kneeling, 1926. Courtesy of George Eastman House, International Museum of Photography and Film (C) The Nickolas Muray Estate. référence à la Sainte Face du Christ des icônes médiévales. Robeson n'est pas seulement statufié, son visage acquérant le statut de relique érigée en altérité absolue, l'exaltation de son corps passe encore par le geste du sculpteur associé au mythe de Pygmalion à travers un jeu de renvoi intertextuel au poème Red Roses for Bronze de H.D 24. Si l'on met l'accent sur la sensualité de son corps plutôt que sur la référence à l'iconographie médiévale, la mise en scène de Robeson dans Borderline évoque les photographies de Nickolas Muray ${ }^{25}$ prises en 1925 et 1926: dans les séries Nude Standing et Nude Kneeling, Robeson pose nu dans des postures qui évoquent la sculpture hellénique, tout en composant une figure féminisée. Ses poses, étendu sur son lit ou se tenant face à une chute d'eau avec Eslanda, ainsi que les mouvements de la caméra parcourant son corps le construisent en objet du désir marqué par une certaine passivité.

Un dernier détour par un récit à clef de H.D., une fiction autour du tournage de Borderline intitulée Two Americans, permet de corroborer cette hypothèse de lecture. H.D., en mettant en scène les projections fantasmatiques qu'inspire Robeson, s'identifie au chanteur noir et au personnage qu'il joue dans Borderline, tous deux partageant la même américanité. Elle le présente comme une divinité archaïque, une idole primitive qui se laisse porter par ses pulsions:

Ce n'était pas un Christ noir. C'était un symbole plus ancien, moins sophistiqué. II représentait Dionysos tel que Nietzsche s'est si vaillamment attaché à le définir [...]. (H.D. 1987: 58, notre traduction)

Dans Borderline, le portrait psychologique de Robeson est complexe, se déployant à la fois sur un plan dramatique, racial, psychanalytique et social. II repose cependant fondamentalement sur le fantasme de l'avènement d'une altérité inaliénée, autrement dit sur un «continent noir» qui définit la sexualité féminine selon Freud ${ }^{26}$. Néanmoins, Borderline instille le trouble dans la définition des races, des sexes et de la normalité psychique en désagrégeant la notion même de «frontière", devenue poreuse et franchissable dans les deux sens.

\section{Body and Soul, ou le détournement de la figure de Robeson dans les pièces de O'Neill}

La figure du Noir qu'interprète Robeson dans Body and Soul (1925) est négative, celui-ci jouant deux personnages opposés, à savoir un révérend (qui s'avère être un détenu évadé) abusant de la crédulité de ses fidèles et un honnête jeune homme de la classe moyenne. Body and Soul constitue, à un premier niveau d'analyse, une attaque frontale contre la corruption de l'Église afro-américaine - ce qui explique en partie l'hostilité de la presse afro-américaine à sa sortie (Bowser et Spence 2000: 191-194, 196-200). Mais, à un second niveau de lecture, se joue dans le film de Micheaux "une critique noire de l'idéologie raciale blanche - des stéréotypes blancs et des tropes narratifs" du cinéma hollywoodien, comme le démontre de façon convaincante l'historien du cinéma Charles Musser (Musser 2001: 102). Micheaux s'approprie dans ce film trois pièces à succès d'auteurs blancs sur la communauté afro-américaine avec Robeson dans le rôle-titre, à savoir Roseanne de Nan Bagby Stephen, The Emperor Jones et All God's 


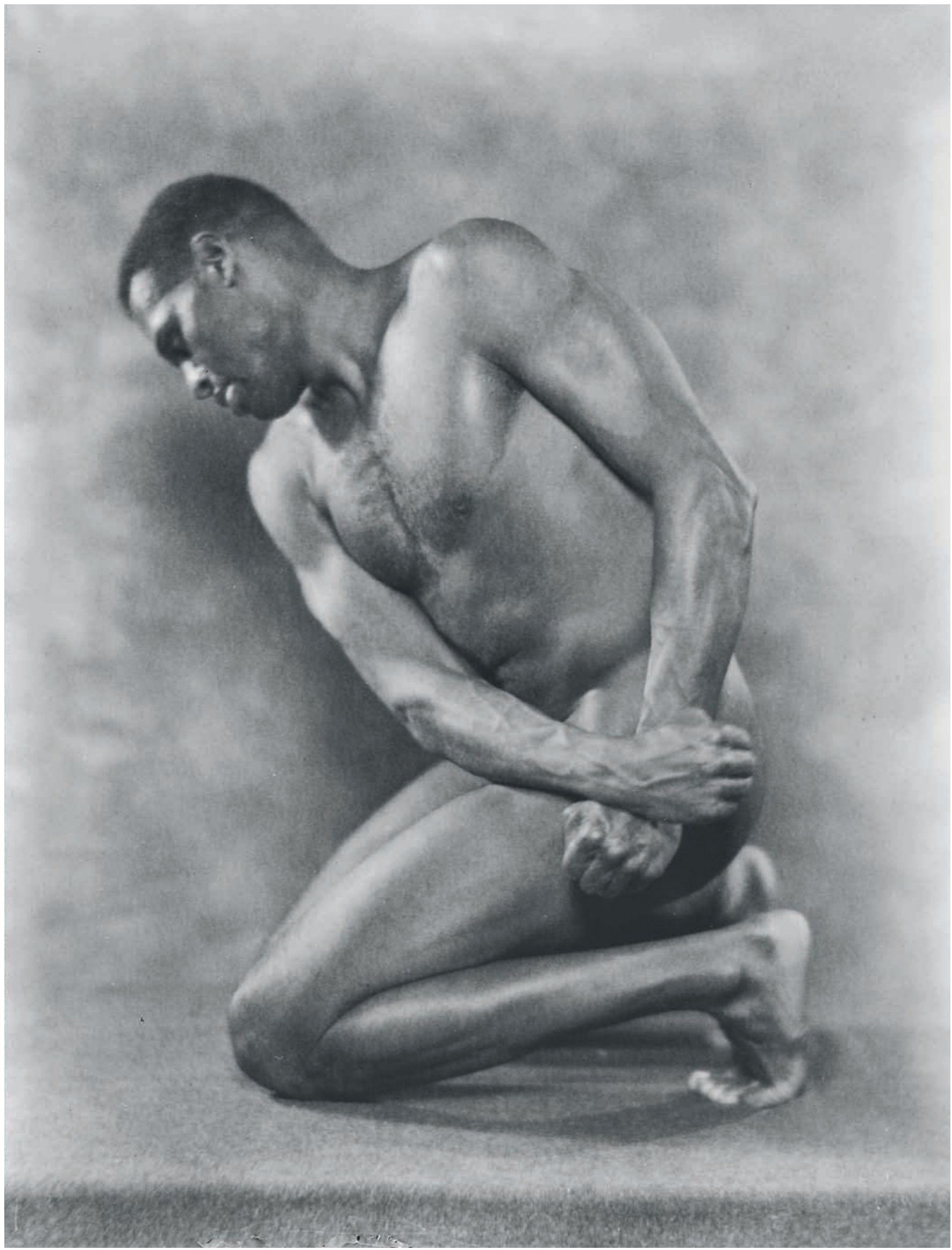




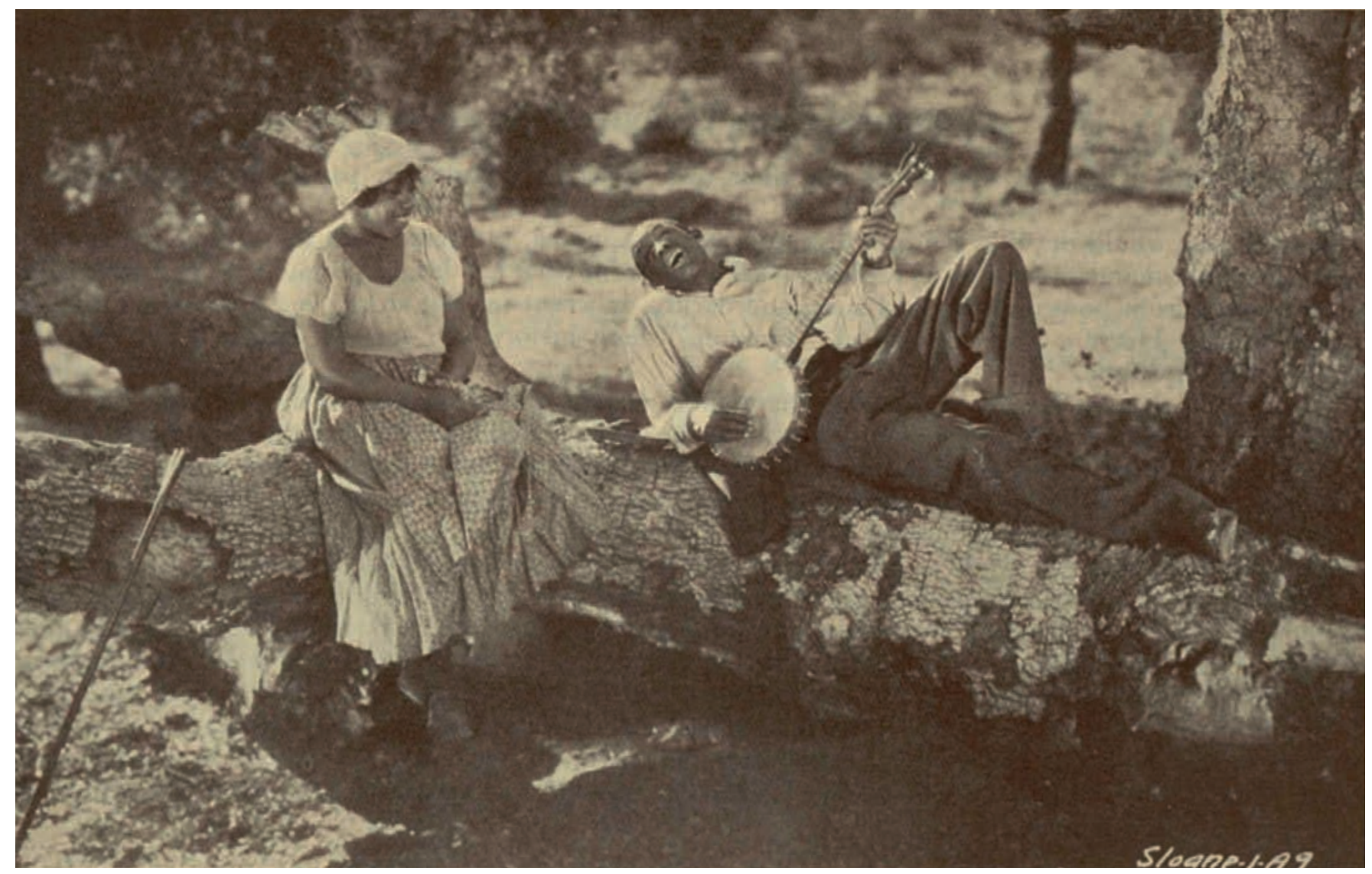

fig. 10

Nancy Cunard (éd.), Negro

Anthology, 1934, p. 337.

Illustration de l'article de

Kenneth Macpherson

montrant Stepin Fetchit dans

le film Hearts of Dixie (1929). 
Chillun' Got Wings de O'Neill. II inverse ainsi la perspective d'écrivains blancs sur la communauté noire en stigmatisant leurs stéréotypes modernistes - ce qui a pour effet de le priver de la sympathie des protagonistes de la Renaissance de Harlem, dont Robeson est un éminent représentant, et de celle des critiques qui défendent une représentation «authentique» de race ${ }^{27}$. Comme le souligne Musser, c'est l'acteur Robeson qui permet de faire le lien entre ces différentes pièces, et non l'auteur ou le réalisateur Micheaux ${ }^{28}$. Le trope de l'inversion, qui est au cœur des films de Micheaux, est redirigé dans Body and Soul contre le personnage primitiviste que Robeson campe sur scène: le mécanisme d'intertextualité évoqué précédemment pour Within Our Gates porte ici sur des rôles de théâtre plutôt que sur des textes ou des scénarios. Body and Soul se caractérise par la réflexivité de sa mise en scène: le film reproduit et met en abyme les stéréotypes raciaux qui surdéterminent les personnages de théâtre interprétés par Robeson, malgré la volonté d'émancipation et le militantisme de l'acteur.

Body and Soul ne se réduit pas à une parabole sur la mystification que représente l'acte de foi inconditionnel dans l'Église (malgré le personnage caricatural de la mère de l'héroïne, qui pousse sa fille dans les bras du révérend avant de la renier - confondant la conversion et le viol, comme l'explicite le film à l'occasion d'une séquence mémorable ${ }^{29}$ ). La dualité du personnage joué par Robeson est accentuée par l'ambivalence des modes d'énonciation dans Body and Soul, oscillant entre rêve et souvenir, cauchemar et réalisme, brouillant les références du film (qui est scindé en deux parties distinctes, la seconde empruntant ses codes au réalisme documentaire). L'artifice énonciatif du rêve dont s'éveille in extremis la mère de l'héroïne, qui a condamné à mort sa fille en refusant de croire en la culpabilité du révérend, marque bien la duplicité de la représentation qui repose sur la duplication et l'inversion des stéréotypes de race. La scène du prêche de la «vallée des ossements» est exemplaire à cet égard, donnant lieu à une série d'inversions à partir du negro spiritual de James Weldon Johnson ${ }^{30}$. Robeson entonne avec conviction un sermon face à une communauté afroaméricaine crédule: premier principe d'inversion, il entrecoupe son prêche d'une série de rasades d'alcool ingurgitées à la dérobée alors qu'il évoque des ossements desséchés; seconde inversion, le public écoute le prêche avec emportement, suivant les stéréotypes du Noir naï et de la mammy niaise; premier renversement de l'inversion, la mère de l'héroïne interrompt le sermon et dénonce le rôle du révérend dans la mort de sa fille, et celui-ci est dès lors pris à partie par la foule; second renversement, l'homme d'Église obtient le pardon de la mère et - dernière volte-face - tue le fils qui voulait venger le décès de sa sœur. Body and Soul, comme le signifie la dualité de son titre, "corps et esprit », met en jeu une dynamique de parallélismes et de comparaisons qui emprunte parfois la structure du montage parallèle de situations opposées. L'image publique de Robeson en tant qu'homme de théâtre est au centre du véritable procès d'intention intenté par Micheaux: la double conscience porte en fin de compte sur l'opposition entre l'élite noire cultivée de Harlem, à travers le personnage du révérend, et la classe moyenne afro-américaine, idéalisée par Micheaux et réinscrite dans le film à travers le personnage positif que joue également Robeson, jeune homme honnête et entreprenant qui épouse l'héroïne.
27. On peut ainsi lire dans The Chicago Defender: "Quelle excuse un homme de notre race peut-il invoquer quand il dépeint les Noirs comme des brutes qui violent nos propres femmes? Devrions-nous nous asseoir et regarder calmement cette production qui nous dénigre en tant que Nègres? Quant aux mérites de cette production: elle n'en a aucun. La chaire et l'écran exercent l'influence la plus profonde sur le public, mais qui à présent, parmi nos prêcheurs et nos producteurs, plantera la bonne graine dans ce sol fertile? En vue de gagner rapidement quelques dollars, ils trahissent leur propre race, et déshonorent même leur métier. Enfin, j'exprime ma reconnaissance aux quelques rares artistes, prêcheurs et producteurs qui respectent leur propre race et qui continueront à œuvrer pour son intérêt sans prendre en considération une ignominie telle que Body and Soul. " (William Henry, "Correcting Oscar Micheaux", The Chicago Defender, 22 janvier 1927, cité par Regester 2001: 46 , notre traduction)

28. "Ici, le déplacement opère sur un double tableau. D'une part, Micheaux indifférencie et réunit les pièces, car elles partagent la même perspective raciale, indépendamment des auteurs. D'autre part, Robeson endosse le rôle qui est traditionnellement joué par l'auteur - une assignation peu flatteuse étant donné le jugement critique que Micheaux exerce envers ces pièces. " (Musser 2001 : 101 , notre traduction)
29. À l'occasion d'une promenade à cheval, le révérend viole la jeune fille tous deux se réfugiant dans une maison abandonnée lorsque la tempête fait rage (séquence rapportée en flash-back dans la dernière partie du film). Après avoir dérobé l'argent de la mère de l'héroïne caché dans une bible, I'homme d'Église affirme posément qu'un combat s'est engagé pour la sauvegarde de l'âme de la jeune fille, qui s'est conclu par sa conversion.

30. Poète, éditeur, compositeur et secrétaire du NAACP, James Weldon Johnson est une figure de premier plan de la Renaissance de Harlem. 
31. Le film s'ouvre sur un intertitre qui présente le personnage du révérend Isiaah T. Jenkins (dit Jeremiah le Sauveur), interprété par Robeson; il est suivi d'une coupure de presse qui rend compte de l'arrestation d'un prisonnier qui s'est évadé lors de son extradition à Londres, sous la fausse identité du révérend Jenkins. Micheaux fait ici allusion à Robeson, qui interprète The Emperor Jones sur les scènes de Londres à la place de Charles Gilpin qui endossait ce rôle depuis les premières représentations de la pièce et a toujours cherché à contrecarrer le caractère raciste des personnages qu'il jouait. Apparemment, Robeson a compris et peu goûté l'allusion, à la différence du public de Body and Soul qui a rejeté le film sans percevoir cette dimension satirique et polémique. Voir Musser 2001: 113-115.
L'enjeu de Body and Soul, difficilement appréhendable en 1925, consiste à présenter une image critique des distorsions à l'œuvre dans la représentation des Noirs en s'appuyant sur un acteur charismatique qui incarne des rôles construits par des dramaturges blancs. La dualité théorisée par Du Bois est ici figurée littéralement à l'écran à travers un acteur qui se dédouble en deux faces opposées. Par métonymie, l'homme d'Église représente le colon, la ségrégation et sa dynamique d'oppression. Ces références proliférantes qui mélangent simple reprise, critique radicale de la citation et pastiche, sans qu'il soit toujours possible de les démêler, n'a pu que provoquer l'incompréhension du public - et l'hostilité de Robeson. Pourtant, Body and Soul pourrait aujourd'hui être compris comme un film de l'inversion de l'inversion, du renversement au carré; c'est-à-dire comme une duplication incessante et vertigineuse des stéréotypes de race jusqu'à leur négation, constituant ainsi un document infalsifiable sur la double conscience de l'Afro-Américain que Du Bois qualifiait en ces termes:

Chacun sent constamment sa nature double, un Américain, un Noir; deux âmes, deux pensées, deux luttes irréconciliables; deux idéaux en guerre dans un seul corps noir, que seule sa force inébranlable prévient de la déchirure. [...] L'histoire du Noir américain est l'histoire de cette lutte - de cette aspiration à être un homme conscient de lui-même, de cette volonté de fondre son moi double en un seul moi meilleur et plus vrai. (Du Bois 2007 : 11)

\section{Distorsions du "nouveau Nègre" et mise en abyme des stéréotypes raciaux à l'écran}

Le cinéma, du moins lorsqu'il emprunte le registre de la fiction, repose sur la transposition à l'écran d'un ensemble de représentations. On a donc tenté de suivre et de retracer ici une série de projections et de représentations surdéterminées en confrontant le postulat d'un cinéma authentique de race à la figure et aux rôles de Robeson dans deux films qui se situent en marge des réseaux de production et de diffusion dominants. Dans Borderline comme dans Body and Soul, la représentation s'inscrit à la puissance seconde: figuration de représentations, images dédoublées et en miroir - qu'il s'agisse de distorsions primitivistes d'une altérité fantasmée ou d'un processus infini de mise en abyme de stéréotypes raciaux. Macpherson, Bryher et H.D. cherchent à valoriser l'image du nouveau Nègre en proposant une image filmique positive de Robeson (figure héroïque, objet du désir, centre d'attraction des regards). Ils font appel à lui précisément en tant qu'acteur de théâtre charismatique - le couple Robeson tourne Borderline en dix jours, entre deux représentations de la pièce de O'Neill The Emperor Jones. Micheaux, à l'inverse, déconstruit l'image de scène de Robeson en reproduisant des stéréotypes qui auraient dû s'effondrer d'eux-mêmes sous le regard critique d'un public averti (malgré les clins d'œil et les allusions, il semblerait que la dynamique intertextuelle de Body and Soul n'ait pas été entendue à l'époque de sa première diffusion ${ }^{31}$ ). S'inscrivant dans la postérité du Blackface, à laquelle Micheaux recourra à nouveau dans ses films sonores, Body and Soul tend à déformer les clichés en les reproduisant de façon outrancière. 
Entre Borderline et Body and Soul, si l'on consent à inverser l'ordre chronologique, on évolue d'une promotion du Noir en tant qu'Autre (le dark continent dont parlait Freud) à l'appropriation de clichés qui se déconstruisent dans leur itération - suivant le mode d'énonciation ambivalent du pastiche, que Fredric Jameson oppose à la parodie et à la satire (Jameson 1983: 111-125).

Université de Lausanne - École cantonale d'art de Lausanne francois.bovier@unil.ch 
Bibliographie

\section{Albera, François}

1994 Autour de Lev Koulechov. Vers une théorie de l'acteur. Lausanne, L'Âge d'Homme.

\section{Baker, Houston A.}

1987 Modernism and the Harlem Renaissance. Chicago, University of Chicago Press.

\section{Bogle, Donald}

1989 Toms, Coons, Mulattoes, Mammies and Bucks: An Interpretative History of Blacks in American Films. New York. Continuum.

\section{Bovier, François}

2009 H.D. et le groupe Pool: des avant-gardes littéraires au cinéma "visionnaire». Lausanne, L'Âge d'Homme.

\section{Bowser, Pearl et Spence, Louise}

2000 Writing Himself into History: Oscar Micheaux, His Silent Films, and His Audiences. New Brunswick, Rutgers University Press.

\section{Cripps, Thomas}

1977 Slow Fade to Black: The Negro in American Film, 1900-1942. New York, Oxford University Press.

\section{Cunard, Nancy}

1933 «Scottsboro", Close Up X (3), p. 274-278.

\section{Cunard, Nancy (éd.)}

1934 Negro Anthology. Londres, Wishart \& Co (rééditions abrégées: Ford, Hugh [éd.], Negro: An Anthology, Frédérick Ungar Publishing Co., 1970; New York-Londres, Continuum, 1996/2002)

\section{Debo, Annette}

2001 "Interracial modernism in avant-garde film: Paul Robeson and H.D. in the 1930 Borderline ", Quarterly Review of Film and Video XVIII (4), p. 371-383.

2012 The American H.D. lowa City, University of lowa Press.

Paul Robeson et la représentation des Noirs dans le cinéma de I'entre-deux-guerres Par François Bovier

\section{Dismond, Geraldyn}

1929 "The Negro actor and the american movies ", Close Up V (2) p. $90-97$.

\section{Duberman, Martin Baum}

1988 Paul Robeson. Knopf New York.

\section{Du Bois, William Edward Burghardt}

2007 Les Âmes du peuple noir. Paris, La Découverte, 2007, traduit par Magali Bessone [première publication: The Souls of Black Folk. Chicago, A.C. McClurg and Co, 1903].

\section{Everett, Anna}

2001 Returning the Gaze: A Genealogy of Black Film Criticism 1909-1949. Durham, Duke University Press.

\section{Flam, Jack et Deutch, Miriam (éd.)}

2003 Primitivism and Twentieth Century Art. A Documentary History. Berkeley, University of California Press.

\section{Foner, Philip S. (éd.)}

1978 Paul Robeson Speaks. Writings, Speeches, Interviews, 1918-1974. Secaucus, Citadel Press.

\section{Freud, Sigmund}

1969 "La morale sexuelle "civilisée" et la maladie nerveuse des temps modernes", La Vie sexuelle. Paris, PUF, traduit par Denise Berger [première publication: "Die "kulturelle" Sexualmoral und die moderne Nervosität », 1908].

1994 La Question de l'analyse profane. CEuvres complètes, XVIII, 1926-1930. Paris, PUF, traduit par Janine Altounian, André Bourguignon, Pierre Cotet et Alain Rauzy [première publication Die Frage der Laieanalyse, 1926].

\section{Gaines, Janes}

$\mathbf{2 0 0 1}$ «Within Our Gates: From race melodrama to opportunity narrative ", in Pearl Bowser, Jane Gaines et Charles Musser (éd.) Oscar Micheaux and His Circle: African-American Filmmaking and Race Cinema of the Silent Era. Bloomington, Indiana University Press.

\section{Green, J. Ronald}

1993 "'Twoness' in the Style of Oscar Micheaux », in Manthia Diawara (éd.), Black American Cinema. New York, Routledge.

2004 With a Crooked Stick: The Films of Oscar Micheaux. Bloomington, Indiana University Press.

H.D.

1983 Red Roses for Bronze, in Louis L. Martz (éd.), Collected Poems, 1912-1944. New York, New Directions [première publication: Londres, Chatto and Windus, 1931].

1987 "Two Americans", New Directions 51 [première publication: H.D., The Usual Star. Dijon, Darantière, 1930].

2006 Borderline. Genève, HérosLimite-Fondation Vevey, Ville d'Images, traduit par Monique Rival [première publication: H.D., Borderline: A Pool Film with Paul Robeson. Londres, Mercury Press, 1930; repris in James Donald, Anne Friedberg et Laura Marcus (éd.), Close Up 1927-1933. Cinema and Modernism. Princeton, Princeton University Press, 1998]

\section{Herring, Robert}

1929 "Black shadows" Close Up V (2), p. 97-104

\section{Hutchinson, George}

1995 The Harlem Renaissance in Black and White. Cambridge, Belknap et Harvard University Press.

\section{Jameson, Fredric}

1983 "Postmodernism and consumer society ", in Hal Foster (éd.), The Anti-aesthetic: Essays on Postmodern Culture. Washington, Bay Press.
Jonas, Gilbert $\mathbf{S}$.

2005 Freedom's Sword: The NAACP and the Struggle against Racism in America, 1909-1969. New York, Routledge.

\section{Koulechov, Lev}

1994 L'Art du cinéma et autres écrits. Lausanne, L'Âge d'Homme.

\section{Lewis, David Levering}

1981 When Harlem Was in Vogue. New York, Alfred Knopf.

\section{Locke, Alain (éd.)}

1925 The New Negro: An Interpretation. New York, Albert and Charles Boni [réédition: New York, Simon \& Schuster, 1992].

\section{Macpherson, Kenneth}

1929 "As is », Close Up V (2), p. 85-90.

1934 "A Negro Film Union - Why not? ", in Nancy Cunard (éd.) 1934, p. 335-338.

\section{Macpherson, Kenneth et Bryher, Winifred (éd.)}

1929 Close Up. A Magazine Devoted to the Art of Films V (2) [réédition: New York, Arno Press, 1971].

\section{McGilligan, Patrick}

2007 Oscar Micheaux: The Great and Only. New York, Harper Collins.

\section{McGinty, Doris Evans et Shirley, Wayne}

1998 "Paul Robeson, musician in Jeffrey C. Stewart (éd.), Paul Robeson: Artist and Citizen. New Brunswick-Londres, Rutgers University Press et The Paul Robeson Cultural Center.

\section{Mercurius}

1930 "Act, fact and abstraction: Borderline. A Pool film ", Architectural Review LXVIII (418). 


\section{Musser, Charles}

1998 «Troubled relations: Pau Robeson, Eugene O'Neill, and Oscar Micheaux", in Jeffrey C. Stewart (éd.), Paul Robeson Artist and Citizen. New BrunswickLondres, Rutgers University Press et The Paul Robeson Cultura Center.

2001 "To redream the dreams of white playwrights: Reappropriation and resistance in Oscar Micheaux's Body and Soul ", in Pearl Bowser Jane Gaines et Charles Musser (éd.), Oscar Micheaux and His Circle: African-American Filmmaking and Race Cinema of the Silent Era. Bloomington, Indiana University Press.

\section{Regester, Charlene}

2001 "The African-American press and race movies, 1909-1929 ", in Pearl Bowser, Jane Gaines et Charles Musser (éd.), Oscar Micheaux and His Circle: African-American Filmmaking and Race Cinema of the Silent Era. Bloomington, Indiana University Press.

\section{Richardson, Dorothy}

1929 "Continuous performance: Dialogue in Dixie ", Close Up IV (3), p. 211-218.

\section{Rubin, William (éd.)}

1984 Primitivism in 20th Century Art: Affinity of the Tribal and the Modern. New York, Musem of Modern Art.

\section{Spivak, John}

1932 Georgia Nigger. New York, Brewer, Warren and Putnam.

\section{Stewart, Jeffrey C.}

1998 "The Black body: Paul Robeson as a work of art and politics ", in Jeffrey C. Stewart (éd.) Paul Robeson: Artist and Citizen. New Brunswick-Londres, Rutgers University Press et The Paul Robeson Cultural Center.

\section{Walton, Jean}

1998 " Nightmare of the uncoordinated white-folk: Race, psychoanalysis, and H.D.'s Borderline ", in Christopher Lane (éd.), The Psychoanalysis of Race. New York, Columbia University Press.

1999 «White neurotics, Black primitives, and the queer matrix of Borderline ", in Ellis Hanson (éd.), Out Takes: Essays in Queer Theory and Film. Durham, Duke University Press.

\section{Weinberg, Herman G.}

1933 "The Emperor Jones", Close Up X (4), p. 351-352.

\section{Willis, Deborah}

1998 "The Image and Paul Robeson ", in Jeffrey C. Stewart (éd.), Paul Robeson: Artist and Citizen. New Brunswick-Londres, Rutgers University Press et The Paul Robeson Cultural Center.

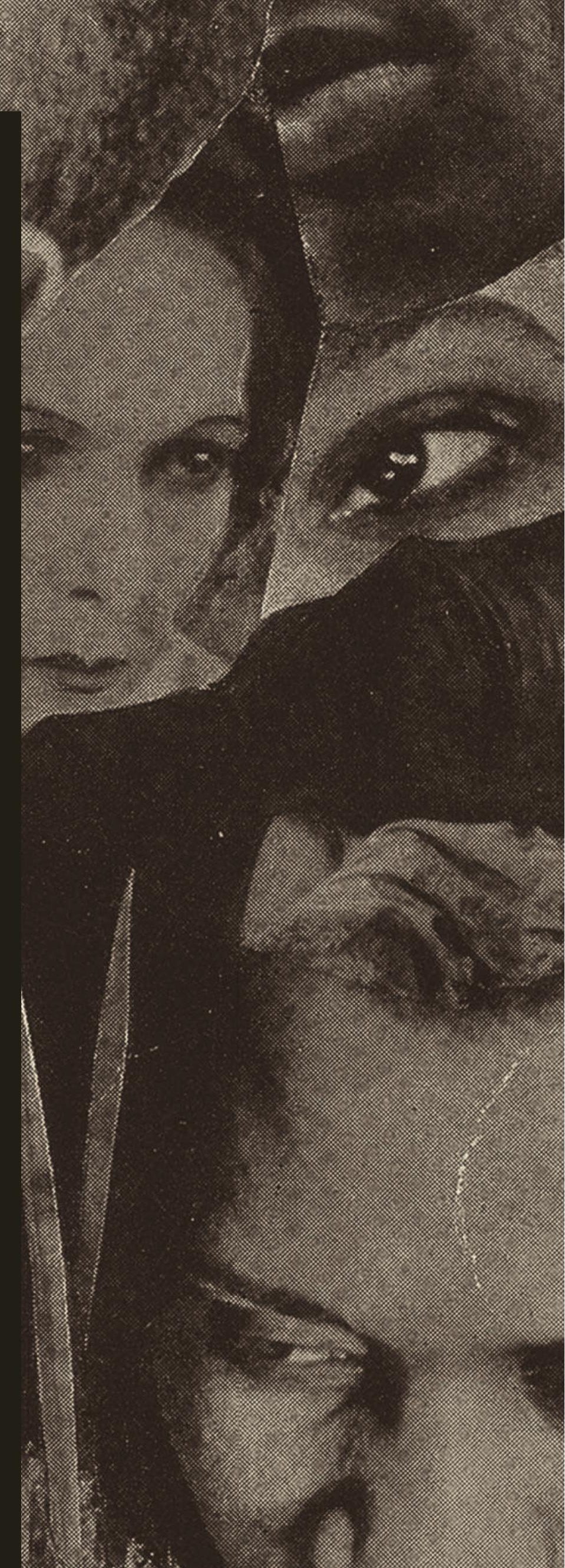

ci-contre

H. D. (Hilda Doolittle), Borderline, Pool film, avec Paul Robeson, 1930. Yale Collection of American Literature, Yale University Beinecke Rare Books \& Manuscript Library, Za D721 930B () H.D. Estate. 